\title{
Labyrinthe
}

$33 \mid 2009$ (2)

"Patates chaudes " : poétique, savoirs, politique

\section{Poétiques de l'histoire}

\section{Hayden White}

Traducteur : Laurent Ferri

\section{(2) OpenEdition}

Journals

Édition électronique

URL : http://journals.openedition.org/labyrinthe/4029

DOI : $10.4000 /$ labyrinthe.4029

ISSN : 1950-6031

Éditeur

Hermann

Édition imprimée

Date de publication : 23 octobre 2009

Pagination : 21-65

ISBN : 978-2-7056-6976-8

Référence électronique

Hayden White, «Poétiques de l'histoire », Labyrinthe [En ligne], 33 | 2009 (2), mis en ligne le 23 octobre 2011, consulté le 03 mai 2019. URL : http://journals.openedition.org/labyrinthe/4029 ; DOI : 10.4000/ labyrinthe.4029

Propriété intellectuelle 


\title{
POÉTIQUES DE L'HISTOIRE
}

\author{
Hayden White \\ Traduit de l'anglais par Laurent Ferri ${ }^{1}$
}

Ce livre, qui est une histoire de la conscience historique dans l'Europe du XIX ${ }^{\mathrm{e}}$ siècle, se veut aussi une contribution à la réflexion actuelle sur le problème de la connaissance historique. S'il rend compte de l'évolution de la réflexion historique pendant une période donnée, il offre de surcroît une théorie structurelle d'ensemble du mode de pensée que l'on qualifie d' « historique ».

Que veut dire « penser historiquement »? Qu'est-ce qui différencie la méthode historique des autres types de recherche? Certes, ces questions ont été débattues tout au long du XIX ${ }^{\mathrm{e}}$ siècle par les historiens, les philosophes et les théoriciens de la société; toutefois, l'idée a longtemps prévalu que l'on pouvait y répondre sans équivoque. On considérait que l'histoire représentait un régime spécifique d'existence, la « conscience historique » un régime spécifique de pensée, et la « connaissance historique » un domaine à part dans l'éventail des sciences humaines et physiques.

$\mathrm{Au} \mathrm{XX}^{\mathrm{e}}$ siècle, toutefois, ces questions ont été abordées dans un état d'esprit différent: à l'assurance a succédé une forme d'inquiétude; on a commencé à se demander s'il était possible d'être aussi catégorique. Des penseurs d'Europe continentale aussi différents que Valéry, Heidegger, Sartre, Lévi-Strauss, Foucault, ont plus que mis en doute l'existence d'une « conscience historique » dotée de vertus particulières ${ }^{2}$; ils n'ont cessé de souligner le caractère fictif des reconstitutions historiques, et ils ont porté un coup sérieux aux prétentions « scientifiques » des historiens. Dans le même temps, des philosophes anglo-américains ont produit un vaste corpus bibliographique concernant le statut épistémologique et la fonction culturelle de la réflexion historique: dans l'ensemble, leurs

\footnotetext{
1. Édition originale: Metahistory, the historical Imagination in nineteenth-century Europe, Baltimore, Johns Hopkins University Press, 1973, p. 1-42.

2. Voir mon article, «The Burden of History ", History and Theory, 5, n 2, 1966, p. 111-34, pour un examen des motifs de cette révolte contre la conscience historique. Pour les plus récentes manifestations de ce phénomène, voir Claude Lévi-Strauss, La Pensée sauvage, Paris, Plon, 1962, et Le Cru et le cuit, Paris, Plon, 1964. Deux ouvrages de Michel Foucault peuvent également être consultés avec profit: Les Mots et les choses, Une archéologie des sciences humaines, Paris, Gallimard, 1966 et L'Archéologie du savoir, Paris, Gallimard, 1969.
} 
travaux nous invitent à remettre en cause le statut de l'histoire comme travail véritablement scientifique ou artistique ${ }^{1}$. Dans les deux cas, on a commencé à se dire que la fameuse " conscience historique », dont les Occidentaux s'étaient fait une gloire depuis le début du XIX ${ }^{e}$ siècle, fut tout au plus le fondement théorique d'une position idéologique, celle depuis laquelle l'Occident a défini sa relation aux cultures et aux civilisations qui l'ont précédé, mais aussi aux sociétés qui lui étaient contemporaines historiquement et contiguës géographiquement ${ }^{2}$. Pour aller vite, il est possible d'y voir un préjugé typiquement occidental, qui a permis rétrospectivement de légitimer la supériorité de la société moderne et industrielle.

Ma propre analyse, qui porte sur la structure profonde de l'imaginaire historique du XIX ${ }^{\mathrm{e}}$ siècle en Europe, entend ouvrir de nouvelles perspectives sur la nature et la fonction de la connaissance historique. J'étudierai à la fois les travaux des grands noms de l'historiographie et ceux des grands philosophes de l'histoire au XIX ${ }^{\mathrm{e}}$ siècle. Un premier objectif général est de définir des caractéristiques permettant de regrouper au sein d'une même « famille » différentes conceptions du processus d'écriture historique, sous la plume de narrateurs classiques. Je me donne aussi pour but d'identifier les différentes théories de légitimation de l'histoire produites par les philosophes. Afin d'y parvenir, je pars d'une définition de l'œuvre historique qui n'est guère contestable: une structure verbale sous la forme d'un récit en prose qui prétend re-présenter, modéliser, et emblématiser des structures et des processus révolus, dans le but de faire comprendre le passé3.

\footnotetext{
1. Le fond de ce débat a été bien résumé par Louis O. Mink, «Philosophical Analysis and Historical Understanding ", Review of Metaphysics, 21, n 4, juin 1968, p. 667-98. La plupart des positions défendues sont reprises au sein de 1'ouvrage de William H. Dray (dir.), Philosophical Analysis and History, New York, Harper \& Row, 1966.

2. Michel Foucault, Les Mots et les choses, op. cit., p. 378-385.

3. Naturellement, la plupart des questions controversées que je m'apprête à aborder ici ont déjà fourni la matière d'amples débats, qu'il s'agisse du « réalisme », de la « représentation littéraire », etc. Pour une discussion de ce problème, voir René Wellek, Concepts of Criticism, New Haven et Londres, Yale UP, 1963. En général, je suis sur ce point l'approche historiographique d'Erich Auerbach dans son ouvrage Mimesis : la représentation de la réalité dans la littérature occidentale, Paris, Gallimard, 1968. Pour ce qui est de la question de la représentation « fictive » de la « réalité », elle a été traitée en profondeur, avec une référence particulière aux arts visuels. Pour Gombrich, le réalisme pictural dans l'art occidental trouve son origine dans l'effort des artistes grecs pour donner une traduction visuelle aux récits épiques, dramatiques et historiques des écrivains. Le quatrième chapitre d'Art et Illusion oppose la surdétermination conceptuelle de l'art mythologique du Moyen-Orient et le caractère non
} 
En bon «formaliste », il ne m'intéresse pas de décider que tel historien vaut mieux que tel autre, parce qu'il rend mieux compte d'événements spécifiques ou d'un segment de l'histoire. Je cherche plutôt à comprendre comment ces comptes-rendus sont structurés.

Mon approche implique de se concentrer sur les travaux d'historiens et de philosophes canoniques, ceux qui font encore aujourd'hui figure de modèles reconnus pour l'historien: Michelet, Ranke, Tocqueville, et Burckhardt d'une part; Hegel, Marx, Nietzsche, et Croce d'autre part. Je considère comme hors-sujet la question de savoir lequel de ces penseurs est le plus exact dans son approche de l'étude historique. Leur

\footnotetext{
mythique de l'art de la narration chez les Grecs. Certes, les deux auteurs semblent ne rien avoir en commun : Auerbach est un hégélien, adepte du fameux « ton apocalyptique en philosophie », tandis que Gombrich s'inscrit dans la tradition néo-positiviste (et anti-hégélienne) dont Karl Popper est le plus éminent représentant. Néanmoins, on gagnerait à comparer l'analyse du « réalisme dans l'art occidental » chez Gombrich et le célèbre chapitre d'ouverture de Mimesis, dans lequel Auerbach se livre à une comparaison ligne à ligne des styles de narration du Pentateuque et des récits homériques. En effet, les deux œuvres abordent un problème commun, à savoir la nature « réaliste» de la représentation, qui est aussi le problème de l'historiographie moderne. Aucun de ces deux livres, toutefois, ne livre d'analyse de la notion essentielle de « représentation historique ", même s'il y est question du sens de l'histoire comme aspect central du réalisme dans le domaine des arts. J'ai, en un sens, inversé les termes employés. Au lieu de: « qu'est-ce qui est proprement historique dans l'art réaliste? », je me demande: « qu'est-ce qui est artistique au sein de l'historiographie réaliste? » Pour répondre à cette question, je me suis fortement appuyé sur les travaux de deux théoriciens de la littérature qui ont quasiment achevé des systèmes philosophiques: Northrop Frye, The Anatomy of Criticism : Four Essays, Princeton, Princeton UP, 1957 et Kenneth Burke, A Grammar of Motives, Berkeley, University of California Press, 1969. J'ai également tiré un grand profit de la critique structuraliste française: Lucien Goldmann, Roland Barthes, Michel Foucault et Jacques Derrida. Je tiens à souligner, toutefois, que ces derniers me semblent être par trop prisonniers de stratégies d'interprétation tropologiques, ce en quoi ils ne diffèrent pas de leurs homologues du XIX ${ }^{\mathrm{e}}$ siècle. Foucault, par exemple, ne semble pas conscient du fait que, pour analyser l'histoire des sciences de l'homme, il ne fait que formaliser un espace de figures rhétoriques. Je l'ai indiqué dans mon essai, «Foucault Decoded: Notes from Underground», History and Theory, 12, $\mathrm{n}^{\circ} 1,1973$, p. 23-54. De mon point de vue, toute la discussion sur la nature du « réalisme » dans la littérature se débat dans des contradictions insolubles, à ne pas vouloir définir de manière critique la « réalité » dans sa dimension proprement historique. La tactique habituelle consiste à poser qu'il existe un pôle historique, domaine de l'empirie, par opposition à un pôle mythique, purement conceptuel: le royaume de la fiction se trouverait entre les deux. La littérature est alors considérée comme étant plus ou moins réaliste, en fonction du ratio empirique/conceptuel. Cette tactique est à l'œuvre aussi bien dans Frye que dans Auerbach ou Gombrich, même s'il convient de noter que le premier a reconnu qu'il y avait un problème, dans un texte intitulé « New Directions from Old », dans Fables of Identity, New York, Harcourt, Brace \& World, 1963, qui traite des relations entre histoire, mythe et philosophie de l'histoire. Parmi les philosophes qui ont le mieux traité du « fictif » dans le récit historique, citons W.B. Gallie, Philosophy and the Historical Understanding, New York, Schoken Books, 1968, Arthur C. Danto, Analytical Philosophy of History, Cambridge, Cambridge UP, 1965; Louis O. Mink, « The Autonomy of Historical Understanding » dans William Dray, Philosophical Analysis and History, op. cit., en particulier p. 179-86.
} 


\section{Labyrinthe, $n^{\circ} 33$}

statut en tant que possibles modèles pour la représentation historique ou la formulation de concepts ne dépend pas de la nature des « données » qu'ils ont utilisées à l'appui de leurs généralisations, ou des théories qu'ils ont invoquées, mais plutôt de la cohérence, de l'homogénéité, de la puissance de leurs visions respectives de la discipline historique. C'est pourquoi leurs systèmes ne sauraient être « réfutés » ou « invalidés » sous prétexte que de nouvelles données ont réorienté des recherches ultérieures, ou qu'une nouvelle théorie de l'interprétation est venue transformer les séries d'événements qui forment la trame de leur représentation et de leur analyse. Ce qui, en définitive, fait de ces auteurs des modèles pour la conceptualisation et la narration historiques, c'est la poétique qui, en amont du concept, sous-tend leur compréhension des processus d'écriture de l'histoire. Cette hypothèse sert de point de départ et de justification à toute approche formaliste de la réflexion historique au XIX ${ }^{\mathrm{e}}$ siècle.

Cela étant, il saute aux yeux que tous ces penseurs ont produit des conceptions alternatives et a priori incompatibles des mêmes séquences du processus d'écriture et de métaréflexion historiques. Du simple point de vue de leur structure verbale, ces conceptions présentent des caractéristiques formelles et déploient des appareils conceptuels qui sont radicalement différents. Pour s'en tenir au niveau le plus superficiel, le travail d'un historien est soit de nature diachronique - auquel cas il met l'accent sur les changements et les transformations au sein d'un processus historique; soit de nature synchronique - et il souligne alors les continuités structurelles. Là où certains historiens se donnent pour mission de faire revivre, de manière poétique ou lyrique, «l'esprit particulier » d'une époque, d'autres veulent révéler les « lois » ou les « principes » dont les événements ne sont que la manifestation phénoménale. Autre distinction fondamentale: alors que certains historiens conçoivent avant tout leur œuvre comme une contribution à la compréhension des problèmes et conflits sociaux contemporains, d'autres, qui relèvent pour l'essentiel de la mentalité dite « érudite », n'ont que faire de telles préoccupations « présentistes » : ils essayent plutôt de déterminer à quel point telle période du passé diffère de la leur. Bref, à les considérer comme pures structures verbales, les « histoires » produites par les grands historiens du XIX ${ }^{\mathrm{e}}$ siècle nous mettent en présence de conceptions radicalement différentes du « travail historique »- de ce que doit être l'histoire. 
Afin de nous familiariser avec les différents types de réflexion historique produits par le XIX ${ }^{e}$ siècle, il est nécessaire de préciser ce que pouvait recouvrir idéalement la structure-type du « travail historique ». Une fois que nous disposerons d'une telle catégorie, je définirai une méthode permettant de déterminer quels sont les aspects déterminants ou structurels de chaque œuvre d'historien ou de philosophe de l'histoire. J'évoquerai ensuite la manière dont ces historiens/philosophes ont retravaillé ces catégories, et les ont intégrées dans un certain type de récit à valeur démonstrative; cela devrait me permettre de dresser la liste des changements fondamentaux survenus dans la structure profonde de l'imaginaire historique au cours du XIX ${ }^{\mathrm{e}}$ siècle, et, passant de la structure aux acteurs, d'étudier les caractéristiques stylistiques propres à chaque contributeur au sein de cet univers particulier de discours qu'est l'histoire.

\section{La théorie de l'œuvre historique}

Commençons par la distinction entre les différents niveaux de conceptualisation à l'œuvre dans l'histoire: (1) la chronique; (2) «l'histoire qu'on raconte » [story]; (3) le mode de mise en intrigue [emplotment]; (4) le mode argumentaire, et (5) le mode d'implication idéologique. La chronique et «l'histoire qu'on raconte » contiennent avant tout les « ingrédients primaires » de la narration, bien qu'elles représentent déjà une phase de sélection et d'arrangement des données collectées par laquelle le document brut est rendu vraiment « accessible » à un public particulier. Ainsi conçu, le travail historique représente une tentative d'intermédiation entre ce que j'appellerai le champ historique, le matériau brut, la bibliographie et un public.

Tout d'abord, les éléments collectés dans le champ historique sont agencés sous forme de chronique; on passe de celle-ci au récit lorsqu'il devient clair que ces événements constituent un «spectacle» qui se déroule sous nos yeux, avec un début, un milieu et une fin. Au cours de cette transformation de la chronique en "histoire qu'on raconte", certains événements deviennent des motifs inauguraux, d'autres sont présentés comme des résolutions, d'autres enfin marquent des transitions.

Un événement qui est simplement signalé comme s'étant produit à un certain moment et dans un certain contexte acquiert un statut inau- 
gural; par exemple, «Le roi arriva à Westminster le 3 juin. Là, il fit la connaissance de celui qui devait plus tard lui disputer son trône, bien qu'à l'époque on eût dit qu'ils étaient destinés à devenir les meilleurs amis du monde...» Un motif de transition, en revanche, envoie au lecteur le signal que la résolution est à venir, qui donnera tout son sens à l'événement: «Lors de son séjour à Westminster, le roi fut informé par son conseiller que ses ennemis l'attendaient, et que les chances d'un règlement avantageux pour la couronne étaient bien minces. » Enfin, un troisième type existe, où les événements constituent un dénouement et mettent fin à une situation de tension: « Le 6 avril 1333, eut lieu la bataille de Balybourne. Les forces du roi l'emportèrent, les rebelles furent mis en déroute. Le traité de Howth Castle, le 7 juin 1333, permit certes d'établir la paix dans le royaume, mais il s'agissait d'une paix fragile, qui ne survécut pas aux conflits religieux qui mirent le feu au royaume, sept ans plus tard. » Quand différents événements sont intégrés à une série, le lecteur dispose d'une histoire : la simple chronique des faits s'est muée en un tout diachronique, qu'il peut interroger comme s'il était face à une structure synchronique ${ }^{1}$.

Les histoires s'organisent en séquences événementielles, qui vont d'un point de départ à un point d'arrivée (ou fin temporaire). Les chroniques, elles, ne sont pas tenues d'avoir « une fin ». En principe, elles n'ont pas non plus de « fait inaugural», et « commencent» tout simplement quand le chroniqueur commence à enregistrer les événements; elles ne « culminent » pas dans une résolution, et peuvent durer indéfiniment.

\footnotetext{
1. Les distinctions que j'ai tenté d'établir entre « chronique », « histoire », et « intrigue » ont plus de valeur pour l'analyse des ouvrages historiques que pour l'étude de la littérature de fiction. Contrairement aux ouvrages de fiction, comme le roman, les ouvrages historiques sont constitués d'événements qui existent en dehors de la conscience de l'écrivain. Les événements rapportés dans le roman peuvent être inventés de telle sorte qu'ils ne peuvent pas (ou ne sont pas censés) s'inscrire dans l'histoire (de quelque chose). Cela rend difficile la distinction entre une chronique et une fiction littéraire. En un sens, «l'histoire » des Buddenbrooks dans le roman éponyme de Thomas Mann, est indiscernable de la « chronique » des événements rapportés, encore que l'on puisse opérer une distinction entre la chronique avec ou sans « intrigue ». À la différence de l'écrivain, toutefois, l'historien se trouve face à un véritable chaos d'événements déjà en place; il doit sélectionner les éléments de l'histoire qu'il va raconter. Pour ce faire, il retient certains événements, en exclut d'autres, et aux premiers il accorde plus ou moins d'importance. Ce processus d'exclusion et de hiérarchisation sert la constitution par l'historien d'une intrigue singulière (he emplots his story). Sur la distinction entre « histoire » et « intrigue », voir les essais de Shklovsky, Eichenbaum et Tomachevsky, représentants de l'école formaliste russe, dans Russian Formalist Criticism: Four Essays, Lincoln, University of Nebraska Press, 1965, et Northrop Frye, The Anatomy, op. cit., p. 52-53 et p. 78-84.
} 
Par contraste, dans une « histoire », il est permis de discerner une forme (même si l'impression du lecteur est chaotique); certains événements sont retenus et d'autres ne le sont pas, sans exhaustivité, contrairement à ce qui se passe dans la «chronique des années tant à tant ».

D'aucuns prétendent que l'objectif de l'historien est d'expliquer le passé en « trouvant », « identifiant 》 ou « découvrant » des « histoires » qui étaient enfouies dans les vieilles chroniques; et que la différence entre « l'histoire » et « la fiction » réside dans le fait que l'historien « trouve » ses histoires, alors que l'écrivain de fiction « invente » les siennes. Cette conception de la tâche de l'historien n'éclaire pas notre lanterne. Bien entendu, «l'invention » joue également un rôle dans les activités de l'historien. Le même événement peut être utilisé différemment dans divers récit historique, en fonction du rôle qui lui est assigné au sein de telle ou telle séquence narrative. " La mort du roi » peut constituer le début, la fin, ou tout simplement un épisode de transition dans trois histoires différentes. Pour le chroniqueur, cet événement « arrive », point. Il n'a pas de fonction narrative. L'historien, lui, organise les événements que l'on trouve dans la chronique au sein d'une hiérarchie signifiante. En leur assignant des fonctions narratives, il assure la cohérence d'un ensemble d'événements qui font sens pour le lecteur, à même de reconnaître un début, un milieu et une fin.

L'agencement narratif de certains événements issus de la chronique amène l'historien à se poser un certain nombre de questions avant même de commencer son récit, puis au fur et à mesure de la construction. Un premier groupe de questions portent sur les liens entre les chaînons constitutifs d'une histoire que le lecteur peut suivre: «Qu'est-ce qui s'est passé ensuite? » ou « Comment en est-on arrivé là? », « Pourquoi de cette façon? », ou « Ça s'est terminé comment? ». Ces questions déterminent les stratégies narratives auxquelles l'historien a recours en écrivant son histoire. D'une autre nature sont des questions comme: "Quelle différence? », "Qu'est-ce que cela nous apprend? ». Ces questions ont trait à la structure de l'ensemble des événements censés constituer une histoire; elles justifient le renvoi à d'autres histoires qui pourraient être « trouvées », « identifiées », ou « découvertes » dans la chronique. Il y a plusieurs manières d'y répondre. Je distingue (1) l'explication par le type d'intrigue, (2) l'explication par le type d'argument et (3) l'explication par le type d'implication idéologique. 


\section{Labyrinthe, $n^{\circ} 33$}

\section{Explication par le type d'intrigue}

Lorsque la "signification » de l'histoire est saisie en fonction du genre d'histoire, on a affaire à une explication par type d'intrigue. Si, pour raconter un certain nombre d'événements, l'historien adopte la structure d'une tragédie, il fournit par là même un certain " éclairage "; même chose s'il choisit la forme d'une comédie, etc. On appelle emplotment la manière dont une séquence événementielle, une fois mise en récit, se rattache progressivement à un genre particulier.

Dans le droit fil des théories élabores par Northrop Frye dans son Anatomie de la critique, j'identifierai au moins quatre différents modes de mises en récit: la romance, la tragédie, la comédie, et la satire. Il en existe peut-être d'autres, comme l'épopée, et tout récit historique est de toute façon susceptible de fondre dans un moule commun des passages qui relèvent de modes différents. Toutefois, un historien est obligé d'opter pour un type d'intrigue dominant, et de rattacher son histoire à une forme archétypale. Par exemple, Michelet raconte toutes ses histoires sur un mode romantique, Ranke sur un mode comique, Tocqueville sur un mode tragique, Burckhardt sur un mode satirique, etc.

La structure de l'épopée semble tout indiquée pour la chronique chaque aspect, même le plus « synchronique » ou « structurel », relèvera dès lors d'une certaine logique narrative. Il en va de même pour le mode satirique: il permet à Burckhardt, dont la manière d'écrire l'histoire semble a priori exclure toute stratégie narrative, de « raconter » comme les autres, mais d'une autre façon. En effet, comme Frye l'a montré, la réussite du mode ironique ou satirique (la satire étant à l'ironie ce que la forme est à la fonction) repose sur la manière de déjouer les codes impliqués par les autres modes - romance, comédie, et/ou tragédie ${ }^{1}$.

\footnotetext{
1. Je réalise qu'en faisant mienne la terminologie de Frye, je m'expose à la critique de ceux qui réfutent sa classification, et proposent une taxinomie différente. Mon intention n'est nullement de suggérer que la critique générique de Frye est la seule possible: elle me paraît seulement la plus utile à mon propos. La principale critique adressée à la grammaire des genres de Frye est qu'elle fonctionne bien pour les sous-genres tels que le conte pour enfants ou le roman policier, mais peine à rendre compte de chefsd'œuvre à structure complexe et entrées multiples, tels que Le Roi Lear, À la Recherche du temps perdu, ou Paradise Lost de Milton. Je suis tout à fait prêt à en convenir; d'un autre côté, l'analyse proposée par Frye se prête à l'étude formelle des récits historiques, ne serait-ce que parce les historiens reculent en général devant les constructions trop compliquées dont les romanciers et les dramaturges se font une spécialité. C'est précisément parce qu'il ne raconte pas une histoire "pour le plaisir» (du moins l'affirme-t-il) que l'historien choisit en général les formes les plus conventionnelles. Certes, au XIX ${ }^{\mathrm{e}}$ siècle,
} 


\section{Poétiques de l'histoire}

Pour l'essentiel, la romance met en scène la réalisation de soi du héros transcendant qui affirme son triomphe sur le monde de l'expérience en s'en affranchissant complètement: il en va ainsi dans la légende du Graal, ou dans le récit mythologique, central pour les chrétiens, de la résurrection du Christ. Il s'agit toujours d'un drame de la victoire du bien sur le mal, de la vertu sur le vice, de la lumière sur les ténèbres, et finalement de la faculté humaine de transcender le monde terrestre qui est sa prison depuis la Chute. La satire va dans la direction opposée. Contrairement au drame romantique, qui met en scène une rédemption toujours possible, la satire exprime en fait un écart irrémédiable, la crainte que l'homme non seulement soit prisonnier du monde, mais ne maîtrise pas son destin, puisque sa volonté achoppe toujours sur le constat qu'elle ne saurait triompher de la mort, sombre et implacable ennemi des hommes.

Les deux formes restantes, la comédie et la tragédie, semblent toutefois offrir la possibilité d'une émancipation au moins partielle de la condition humaine, vue comme une déchéance terrestre et un écartèlement permanent. Chacune à leur façon, elles laissent ouverte la possibilité d'une victoire et même d'un triomphe mondains - si provisoires soient-ils. Dans la comédie, l'espoir réside dans la perspective d'une réconciliation des forces en jeu dans le monde naturel comme dans la société. Celle-ci fait l'objet d'une célébration particulièrement festive sous la plume de l'écrivain comique. Dans la tragédie, au contraire, point de célébration d'unité, tout au plus des bouffées d'exaltation illusoire; ce qui est montré, c'est la fatalité de la division entre les hommes, plus terrible encore que l'agôn au début des tragédies antiques. En même temps, la chute finale du protagoniste et l'ébranlement du monde qu'il habite ne sont pas de

\footnotetext{
n'importe quel historien digne de ce nom disposait d'une solide culture littéraire, et connaissait aussi bien les mythes de l'Antiquité que les grands classiques de l'époque moderne. Il était a priori équipé pour repérer et ré-utiliser certaines fícelles narratives. Toutefois, on se méprendrait à imaginer qu'un historien, fût-il aussi subtil que Tocqueville, aurait jamais pu atteindre au même degré de raffinement formel qu'un Shakespeare ou qu'un Racine. De fait, lorsque des historiens comme Burckhardt, Marx, Michelet, ou Ranke emploient des termes comme « tragédie » ou « comédie », c'est souvent avec une compréhension très limitée des enjeux narratifs - il en va différemment de Hegel, Nietzsche, et (jusqu'à un certain point) Croce. L'intérêt de ces philosophes pour l'esthétique leur permit d'approfondir leur compréhension des genres, et de composer des écrits historiques beaucoup plus complexes dans leur construction que ceux des historiens qui, si critiques soient-ils de l'usage de leurs sources, sont en général naïfs devant les enjeux de la narration [naive storytellers]. Voir Northrop Frye, The Anatomy, op. cit., p. 158-238, et Geoffrey Hartman, « Ghostlier Demarcations: The Sweet Science of Northrop Frye, » dans Beyond Formalism : Literary Essays 1958-1970, New Haven et Londres, Yale UP, 1970, p. 24-41.
} 


\section{Labyrinthe, $n^{\circ} 33$}

nature à menacer dans leur être ceux qui survivent à la catastrophe finale. Au contraire, les spectateurs retirent un bénéfice moral de cette mise à l'épreuve. Ils estiment assister de loin au triomphe de l'humanité, au sens d'une loi de l'existence humaine contre laquelle le protagoniste a eu l'impudence de s'élever.

À la fin de la comédie, on assiste à une réconciliation des hommes avec d'autres hommes, ou avec la nature, ou avec la société. Cette dernière s'en trouve assainie et renforcée, puisque des éléments qui semblaient irréconciliables se révèlent, à long terme, pouvoir converger et co-exister harmonieusement. Les « réconciliations » auxquelles nous assistons à la fin des tragédies sont de nature beaucoup plus sombre: l'homme se résigne en fait à sa condition terrestre, qui est de travailler «à la sueur de son front» jusqu'à la fin des temps, et sans possibilité d'y rien changer. La tragédie fixe les limites sur ce qu'il est assurément permis et convenable d'espérer sur terre.

La romance et la satire semblent mutuellement exclusives. La notion même d'une satire romantique paraît une contradictio in terminis. Certes, il est possible qu'une romance ait un fort contenu satirique; mais alors son but serait d'exposer, d'un point de vue ironique, la vanité complète des conceptions romantiques du monde. Si nous cherchons à former d'autres doublets, nous constatons que satire et comédie fonctionnent bien ensemble, de même que satire et tragédie. Mais, ici, il convient de noter que la relation entre le genre de la comédie ou de la tragédie et le mode satirique est différente de celle qu'on obtient en croisant le genre de la romance et les modes comique et tragique. Dans ce dernier cas, « comédie » et « tragédie » ne font que donner une coloration particulière au même mode d'appréhension romantique du monde, pour laquelle on ne saurait minimiser (comme dans la romance " naïve ») les forces qui s'opposent à l'effort de rédemption de l'être humain. La comédie et la tragédie ont en commun cette conscience lucide, même si la première trouve sa résolution dans une réconciliation des forces opposées, tandis que la seconde culmine dans une victoire éclatante des forces qui résistent à l'homme. Ce qui caractérise l'écrivain romantique, c'est qu'il énonce de grandes vérités sur la condition humaine sur fond d'une même structure - son drame est toujours celui de la rédemption, ou le triomphe de la volonté transcendante, même dans l'échec. 


\section{Poétiques de l'histoire}

Par rapport à la romance, à la comédie, et à la tragédie, la satire envisage différemment l'être humain dans ce qu'il peut espérer réaliser et révéler de lui-même. Si tous les genres ont en commun de présupposer une conscience malheureuse, qui ne peut s'adapter au monde tel qu'il est, c'est-à-dire limité et insatisfaisant, la satire se distingue par son ironie. Elle marque en cela une étape dans l'évolution artistique ou littéraire: tout se passe comme si le mode satirique de représentation s'accompagnait de la conviction que le monde est vieux. La satire rejoint la philosophie dans la conscience de sa propre incapacité à produire une image nette de la réalité : elle ne fait qu'ajouter de la grisaille à la grisaille. Ce faisant, elle prépare nos consciences à s'écarter des approches trop conceptuelles et sophistiquées, et annonce le retour à une appréhension mythique du monde.

Aux quatre formes archétypales d'histoire que nous avons distinguées correspondent quatre types d'exposition de la part de l'historien en quête d'effets narratifs. Il est permis d'opposer les récits de type diachronique, du genre de ceux produits par Michelet et Ranke, et les récits de type synchronique voire statique que l'on trouve chez Tocqueville et Burckhardt. Dans Michelet ou Ranke, la dynamique de la représentation est portée par l'idée de transformation structurelle. Chez d'autres historiens, au contraire, ce qui domine est le sens de la continuité structurelle (particulièrement chez Tocqueville) voire de la stase (chez Burckhardt). Toutefois, cette distinction entre représentation synchronique et représentation diachronique de la réalité historique n'induit pas, ni n'exclut, le recours à tel ou tel type de narration ou d'intrigue. Elle signifie simplement que l'accent est plutôt mis sur la continuité, ou plutôt sur le changement au sein d'une représentation d'ensemble du processus historique.

La tragédie et la satire sont favorisées par les historiens qui croient trouver au sein du tourbillon des événements qu'ils cherchent à percer une permanence dans la logique d'enchaînement, et qui discernent dans la chronique l'éternel retour du même. Au contraire, la romance et la comédie insistent sur l'apparition de nouvelles forces ou de nouvelles conditions, bien qu'à première vue les processus historiques semblent immuables dans leur essence, et les seuls changements des altérations formelles. Dans tous les cas, le choix d'un type d'intrigue ne peut manquer d'avoir une incidence cognitive; il informe la manière dont l'historien cherche à expliquer ce qui s'est « réellement» produit. 


\section{Labyrinthe, $n^{\circ} 33$}

\section{Explication par l'argument formel}

Au niveau de conceptualisation auquel il se trouve quand il écrit son intrigue, l'historien s'emploie à expliquer les tenants et les aboutissants de « ce qui s'est passé ». Il a recours pour cela à des arguments de types divers, formel, explicite, ou discursif. En fait, de tels arguments fournissent une explication de ce qui se passe dans le récit historique en recourant à des agencements élevés au rang de « loi de l'histoire». La logique à l'œuvre est nomologico-déductive, ou syllogistique. La majeure est une loi de causalité supposément universelle, la mineure pose les limites dans lesquelles la loi s'applique, et les événements sont en quelque sorte " déduits» de ces deux prémisses. La plus célèbre de ces « lois universelles » se trouve dans Marx; il s'agit de la relation entre superstructure et infrastructure. Cette loi stipule que, chaque fois qu'il se produit une transformation des modes de production qui forment la base de l'édifice social, il s'ensuit nécessairement un changement dans les institutions et les mentalités sociales et culturelles; l'inverse n'est en revanche pas vrai : ainsi, une nouvelle « prise de conscience » ne suffit pas pour entraîner des transformations dans les modes de production. D'autres lois putatives sont invoquées par l'historien, au moins subrepticement, pour expliquer des phénomènes aussi différents que la Grande Dépression ou la chute de l'Empire romain. La nature proverbiale, voire franchement triviale de ces « lois générales » (« la mauvaise monnaie chasse la bonne ", « tout ce qui monte finit par redescendre ») n'empêche pas l'historien d'y recourir, fût-ce sous la forme d'une démonstration apparemment rigoureuse. Voilà qui devrait nous inciter à la plus grande méfiance vis-à-vis de toute explication proto-scientifique de l'histoire, via le recours maladroit aux sciences sociales - auxquelles de telles généralisations sont empruntées.

Il est important de distinguer jusqu'à un certain point: d'une part, la validité des « lois » prouvées de manière logico-déductive; d'autre part, l'effet produit sur le lecteur par tel ou tel type de narration. Toutefois, des formes de récit peuvent avoir sur le lecteur l'effet de " démonstrations ». Par exemple, un récit tragique part du principe que certaines lois régissent la nature humaine et les sociétés; dans la mesure où certaines circonstances sont réunies à un moment et un endroit donnés, on peut expliquer l'enchaînement des événements historiques qui viennent 
nécessairement à se produire, comme pour les phénomènes « naturels ». C'est pourquoi, quant à sa cohérence, la logique de l'historien ne diffère pas fondamentalement de celle du scientifique. Toutefois, je tiens à conserver pour l'instant la distinction entre événement comme séquence narrative et événement comme élément d'une séquence logique. Faisons donc comme si nous accordions du crédit à l'opposition traditionnelle entre « recherche des faits ", d'une part, et mise en récit, d'autre part, qui fonde la prétention de l'historien à produire à la fois de la science et une œuvre artistique. Comme s'il y avait, d'un côté, « les faits » et « la raison des faits », et, de l'autre, un enchaînement logique de ces faits sous la forme d'une narration conduisant d'une situation historique à l'autre par l'effet d'une loi universelle de causalité.

Le hic, c'est que, à la différence des scientifiques, les historiens sont doublement en désaccord: non seulement sur les prétendues lois sociales de causalité qu'ils choisissent d'invoquer pour expliquer une séquence événementielle, mais également sur la question de la forme idoine pour l'explication «scientifique » des faits. On débat depuis fort longtemps de savoir si l'explication scientifique des faits naturels et celle des faits historiques ou sociaux doivent avoir les mêmes caractéristiques formelles. Les lois qui peuvent être invoquées pour expliquer des phénomènes « scientifiques » ont-elles leur équivalent dans le domaine des sciences dites humaines, telles que la sociologie et l'histoire? Prenons l'exemple des sciences physiques: le progrès dans ce domaine est rendu possible par le fait que les membres de la communauté (des) scientifique(s) arrivent à se mettre d'accord, à intervalles réguliers, sur ce qui constitue un problème scientifique, sur la forme que l'explication scientifique doit prendre, et sur le type de données valides et autorisées pour rendre compte scientifiquement de la réalité de ce problème. Parmi les historiens, rien de tel, aujourd'hui comme hier. Il est possible que cet accord impossible ne fasse que refléter le caractère non scientifique de l'entreprise historiographique; quoi qu'il en soit, ce désaccord congénital a d'importantes conséquences pour l'explication par l'historien d'une série de phénomènes proprement historiques. Il n'arrive à certaines conclusions que parce qu'il part de certains présupposés métahistoriques, portant sur la nature même de son activité; telle conception du champ historique entraîne tel type d'analyse historiographique. 
Le conflit sur les niveaux d'interprétation historique porte en fait sur la « véritable» nature de l'entreprise historiographique. L'histoire se trouve toujours dans la situation d'anarchie conceptuelle qui était celle des sciences naturelles au XVI ${ }^{\mathrm{e}}$ siècle, quand il existait autant de conceptions différentes de «l'entreprise scientifique » qu'il y avait de positions métaphysiques. Au XVI ${ }^{e}$ siècle, les différentes définitions de ce que devait être la science reflétaient en fin de compte différentes visions de la « réalité », et les différentes épistémologies générées par ces systèmes de croyance. De même, les disputes sur ce que l'histoire devrait être ne font que traduire des conceptions divergentes de ce qu'est une bonne explication historique et, partant, un bon historien.

Est-il besoin de le préciser? Je ne parle pas ici des types de différends soulevés par les auteurs de comptes-rendus pour des revues professionnelles, quand ils questionnent l'érudition ou la précision de tel ou tel collègue. Je parle ici des problèmes qui se posent lorsque deux chercheurs (ou plus), à niveau d'érudition et de sophistication théorique à peu près égal, parviennent à des interprétations pas nécessairement incompatibles, mais en tout cas divergentes, de la même série d'événements historiques. Alors qu'ils travaillent sur les mêmes événements, comment se fait-il qu'ils apportent des réponses complètement différentes à des questions comme « la Renaissance, rupture ou continuité? ». Ce qui est en jeu ici, c'est bien le concept de réalité historique et la forme de récit historique retenus. J'ai emprunté à Stephen C. Pepper ses quatre grands paradigmes de formulation d'une explication historique: formiste, organiciste, mécaniste, et contextualiste ${ }^{1}$.

\footnotetext{
1. Les remarques formulées à l'encontre de Frye peuvent s'appliquer, mutatis mutandis, à l'analyse des principales formes archétypales de discours philosophique par Pepper. Certes, les œuvres des plus grands philosophes, qu'il s'agisse de Platon, d'Aristote, de Descartes, de Hume, de Kant, de Hegel, ou de Mill, résistent à toute réduction de cet ordre. Elles relèvent à tout le moins de plusieurs paradigmes et ne se laissent enfermer par aucun. En revanche, le système de Pepper est pratique pour rendre compte de systèmes philosophiques plus simples, propres aux historiens quand ils s'expriment comme des philosophes, c'est-à-dire, quand ils font appel à certains théories générales qui se trouvent vérifiées dans l'histoire, mettent au jour les implications éthiques supposée de vérités établies, etc. La plupart des partisans de cette école dépassent rarement le niveau de sophistication philosophique atteint par, disons, Edmund Burke. Le grand historien conservateur avait certainement une « vision du monde », mais on ne saurait appeler ça une « philosophie ». C'est également le cas de la plupart des historiens, Tocqueville inclus. En revanche, les plus grands philosophes de l'histoire ont tendance à parachever en même temps un système philosophique et une vision du monde. En ce sens, ils agissent de manière plus responsable, au plan cognitif, que les historiens qui, la plupart du temps, ne font qu'accepter sans la discuter une certaine vision du monde et la traitent ensuite comme s'il s'agissait d'une position
} 
La théorie formiste vise à identifier des caractéristiques propres des objets d'étude qui « forment» le champ historique. En conséquence, le formiste considère qu'une explication est complète lorsque l'ensemble des objets ont été correctement identifiés, nommés, et que chacun a été décrit dans sa singularité. Ces objets peuvent être aussi bien des individus que des groupes ou des universaux, des entités concrètes que des abstractions. Ainsi envisagée, la tâche d'explication historique consiste à dissiper l'illusion de similitude des objets présents sur le terrain. Lorsque l'historien a établi le caractère unique de chaque entité ou phénomène, il a fourni une explication formiste ${ }^{1}$.

Ce mode d'explication se trouve chez Herder, Carlyle, Michelet, chez les historiens du romantisme et chez les grands narrateurs que sont Niebuhr, Mommsen et Trevelyan. Faire revivre les événements dans leur diversité, leur couleur, leur flamboyance constitue la mission principale de ces historiens. Bien sûr, un historien formiste peut à l'occasion formuler des généralisations sur la nature du processus historique dans son ensemble, ainsi Carlyle lorsqu'il évoque « un trait commun à d'innombrables biographies ». Toutefois, dans la conception formiste, ce

\footnotetext{
philosophiquement établie. Sur les « hypothèses-monde », voir Stephen C. Pepper, World Hypotheses: A Study in Evidence, Berkeley et Los Angeles, University of California Press, 1966, II, p. 141 sqq. 1. Je suis très redevable à Kenneth Burke dans mon usage de sa terminologie pour caractériser ce que j'appelle «le champ historique » préexistant à l'analyse et à la représentation historique. Burke fait l'hypothèse que toute représentation littéraire de la réalité peut faire l'objet d'une analyse à partir de cinq éléments grammaticaux : la scène, l'agent, l'acte, l'action [agency] et le but. Leurs caractéristiques et leur poids relatif en tant que forces causales dans le « drame » révèlent la vision du monde implicite dans chaque représentation de la réalité. Ainsi, un écrivain matérialiste aura tendance à insister sur la «scène » (c'est-à-dire le milieu) afin de faire passer les autres éléments pour épiphénoménaux. Un écrivain idéaliste, au contraire, mettra le but au premier plan et ne fera de la scène elle-même qu'une illusion. Voir Burke, A Grammar, op. cit., p. 3-20. Si elles sont efficaces pour conceptualiser le « champ historique », les théories de Burke le sont moins pour décrire ce que peut faire l'historien une fois que ce champ a été « grammaticalement » caractérisé. De ce point de vue, sa rhétorique des motifs (Rhetoric of Motives, Berkeley et Los Angeles, 1965) et son langage comme action symbolique (Language as Symbolic Action, Berkeley et Los Angeles, 1968), dont l'objectif était d'explorer les dimensions morales de la représentation littéraire pour la première et de fournir une version sécularisée de la pensée médiévale par « analogie » pour le second, restent tristement classiques. Burke a raison de souligner que toutes les représentations littéraires, même "réalistes », sont au fond allégoriques mais sa classification des types d'allégories n'apporte guère plus que les anthropologies symboliques marxiste ou freudienne - qui sont d'ailleurs elles-mêmes « allégoriques ». Du point de vue allégorique, les méthodes d'analyse proposées par Frye s'appliquent donc mieux à l'histoire. La terminologie de Pepper semble mieux décrire l'histoire vue comme un discours assumé de connaissance. Quant à la sociologie de la connaissance de Mannheim, elle est surtout valide pour les histoires considérées comme des traités de morale. Voir un peu plus loin note 1 p. 44.
} 


\section{Labyrinthe, $n^{\circ} 33$}

qui est au cœur de l'explication des événements, c'est bien la spécificité des différents agents historiques, plus que la «scène » sur laquelle ils font irruption.

Pour reprendre la terminologie de Pepper, le formisme « disperse » les données au cours de l'analyse, alors que les paradigmes d'explication organicistes et mécanicistes tendent à les « intégrer ». De ce fait, l'historien formiste brasse davantage et distingue un plus grand nombre de faits particuliers au sein du champ historique; ses généralisations manquent, en revanche, de "précision » - cela est patent chez les romantiques, et chez les "grands raconteurs de l'histoire » en général. Côté pile, le caractère très vivant de leurs reconstitutions; côté face, la vacuité de leurs généralisations.

En comparaison, le modèle « organiciste » est relativement plus « intégrateur » et, partant, plus réducteur. L'historien organiciste tente de rapporter la diversité des différents objets présents dans le champ historique à des logiques d'ensemble. Au cœur de la stratégie organiciste se trouve une métaphysique de la relation microcosme/macrocosme; l'historien qui accepte cette logique est animé par le désir de voir converger les différentes entités en un processus dynamique unifié, de les agréger en sorte que le tout soit supérieur à la somme des parties. Les historiens qui adoptent cette stratégie d'explication, comme Ranke, et la plupart des historiens « nationalistes » du milieu du XIX ${ }^{e}$ siècle (Sybel, Mommsen, Treitschke, Stubbs, Maitland, etc.), ont tendance à structurer leur récit de manière à représenter la cristallisation d'une série d'événements apparemment dispersés, et qui seraient en fait conduits, par la dynamique du récit, à trouver leur place et leur sens dans un processus beaucoup plus ample.

Il est indéniable, comme le fait observer Pepper, que les historiens de cette trempe, de même que les penseurs dialectiques à la Hegel, sont plus soucieux de caractériser l'intégration des faits dans un processus historique que de les décrire dans leur singularité. C'est ce qui donne à leurs arguments un côté très «abstrait» et « déterministe »-puisque tous les événements tendent vers un telos ou une fin. Un historien comme Ranke se gardera bien, naturellement, de spécifier quelle est cette fin de l'Histoire, et le but de la recherche historique consistera précisément pour lui à mettre au jour des structures provisoires d'achèvement, telles que Volk, Nation ou Kultur. Au-delà, on serait de son point de vue dans la pensée religieuse. Ranke est un historien formiste d'un genre 
particulier: certes, il excelle dans la description des événements dans leur singularité; dans le même temps, ses récits trouvent leur cohérence structurelle et formelle dans les explications qu'il donne du processus historique englobant.

De manière caractéristique, quand ils déploient leurs stratégies d'explication, les historiens organicistes évitent de parler de « lois de l'histoire ", au sens où l'on parle de lois physiques selon Newton, de lois chimiques selon Lavoisier, ou de lois biologiques selon Darwin. Ils diraient plutôt que des « principes » ou des « idées » informent les processus historiques, qu'ils soient individuels ou collectifs. Ces « principes » ou ces « idées » préfigurent (au sens à la fois étymologique et chronologique) l'issue vers laquelle tend tout processus historique, plus qu'ils ne l'orientent dans ses moindres agencements, comme c'est le cas chez les historiens d'obédience théologique ou mystique, pour lesquels tout sera interprété comme la manifestation de la volonté de Dieu veillant sur ses créatures. En fait, pour l'historien organiciste, ces « principes directeurs » ne restreignent pas la capacité qu'ont les êtres humains de parvenir à leurs fins dans l'histoire - comme le feraient des « lois » historiques dans un schéma mécaniciste. Au contraire, ils sont autant de garanties de la liberté humaine. Certes, chaque action ne trouve sa place et son sens que rétrospectivement, et à l'intérieur d'un processus historique mis en évidence par l'historien; pour autant, nul fatalisme ne résulte de l'approche organiciste, alors que l'approche strictement mécaniciste ou nomologique incline à un certain pessimisme sur notre capacité à « faire l'histoire ».

La théorie mécaniciste repose, comme la théorie organiciste, sur l'intégration des actions humaines dans un processus d'ensemble, mais de manière réductrice plutôt que synthétique. Pour reprendre les termes de Kenneth Burke, l'historien mécaniciste part du principe que les « actions » ou les « acteurs » identifiés comme historiques font tout au plus apparaître certaines configurations extra-historiques du milieu « là où l'action se passe » dans la narration. Dans le schéma mécaniciste, tout processus historique est déterminé par un certain nombre de lois de causalité mises à jour par l'historien. C'est pourquoi le mécaniciste, qu'il s'agisse de Buckle, de Taine, de Marx, ou même de Tocqueville, étudie l'histoire pour deviner les lois qui la gouvernent, et écrit l'histoire pour exposer, sous une forme narrative, les effets de ces lois. 


\section{Labyrinthe, $n^{\circ} 33$}

Cela étant, les historiens mécanicistes accordent plus ou moins d'importance à la compréhension des lois qui gouvernent l'histoire, et à la détermination de ces lois, lorsqu'ils représentent " ce qui s'est passé " à tel endroit et à tel moment du processus historique. Cependant, dans la mesure où leur enquête est motivée par la découverte de telles lois, les tenants de l'approche mécaniciste, comme les tenants de l'approche organiciste, courent un risque particulier, celui de faire une histoire abstraite. À leurs yeux, les actes individuels sont moins importants que les phénomènes de groupe auxquels ils se rattachent; ces phénomènes de groupe sont eux-mêmes moins importants que les lois qu'ils confirment par leur régularité. En dernier ressort, les tenants de l'approche mécaniciste ne sont satisfaits que lorsqu'ils ont mis au jour les lois qui gouvernent le déroulement de l'histoire, exactement comme les lois de la physique sont censées gouverner la nature. Les données historiques sont ensuite traitées de sorte qu'on ne peut comprendre les processus historiques qu'en fonction de lois préétablies. C'est ainsi que chez Tocqueville, mais aussi chez Buckle, Marx et Taine, les traits propres à une institution, un texte de loi ou une œuvre d'art sont moins importants que les attributs structurants qui en font des manifestations-types; ces dernières ne font qu'attester la permanence de lois, qui expliquent les changements sociaux dans l'histoire occidentale.

Il va de soi que, pour cette raison, l'approche mécaniciste, en dépit de sa précision conceptuelle, ne peut manquer de se voir reprocher son réductionnisme, et sa tendance à l'abstraction - reproches qui, encore une fois, sont également faits aux tenants de l'approche organiciste. D'un point de vue formiste, elle nie la diversité et pour ainsi dire la polychromie des phénomènes historiques considérés individuellement. Toutefois, il n'est nul besoin, pour retrouver un tel sens de la multiplicité et de la couleur, de se réfugier dans «l'impressionnisme » qui caractérise l'explication formiste de l'histoire. On peut préférer se tourner vers la position contextualiste, qui consiste à expliquer et à hiérarchiser les événements historiques selon une logique « fonctionnelle».

Ici, le présupposé de départ est que l'on ne saurait expliquer les événements qu'en les replaçant dans leur contexte, c'est-à-dire en relation avec les autres événements survenus dans le même environnement historique. Comme dans l'approche formiste, l'histoire est conçue comme un spectacle, ou encore une riche tapisserie qui semble à première vue manquer 
de cohérence, et dont la structure est difficile à démêler. Mais, alors que le formiste tend à ne considérer les différentes événements que dans leur singularité, en insistant sur leurs points communs et leurs différences, le contextualiste rend compte de « ce qui s'est passé à un moment donné » en s'attachant avant tout aux relations que des acteurs contemporains, ou des actions simultanées, entretiennent les uns avec les autres.

La nature de cette interrelation a été étudiée par certains philosophes modernes comme William H. Walsh et Isaiah Berlin. On a donné le nom de « colligation » à l'opération descriptive par laquelle une multitude de détails est totalisée en une seule proposition ${ }^{1}$. Appliquée à l'histoire, la colligation consiste à utiliser certains « fils » qui rattachent l'objet d'étude, individu ou institution, à un contexte socioculturel qui définit le «présent» d'une époque. Des exemples de ce type d'explication se trouvent certes en abondance chez tous les historiens dignes de ce nom, d'Hérodote à Huizinga, mais la colligation triompha comme principe d'explication par excellence au XIX ${ }^{\mathrm{e}}$ siècle, dans l'œuvre de Jacob Burckhardt. L'approche contextualiste veut éviter deux écueils : celui de la dispersion (qui menace toujours le formisme) et celui de l'abstraction (vers laquelle se dirigent à la fois l'organicisme et le mécanicisme). Elle s'efforce au contraire d'intégrer les différents phénomènes contemporains dans des courants, ou trends, et de rapporter les différents traits d'une province déterminée de l'histoire à la " physionomie générale d'une époque ». Pour affirmer que différentes manifestations historiques proches dans le temps ont plus qu'un « air de famille », elle a recours tacitement à des règles bien différentes du principe «telles causes, tels effets » postulé par l'approche mécaniciste, ou des principes téléologiques qui fondent l'approche organiciste. Tout se résume aux relations tangibles observées entre divers phénomènes que l'historien pense pouvoir dater et situer précisément, mais dont il ignorera toujours et l'origine matérielle, et la cause finale.

L'historien contextualiste procède, nous dit Pepper, de la façon suivante: un élément trouvé dans le champ historique est isolé comme objet d'étude; il peut s'agir aussi bien d'un phénomène très large comme « la Révolution française », que d'une journée dans la vie d'un individu

1. Voir William. H. Walsh, Introduction to the Philosophy of History, Londres, 1961, p. 60-65; Isaiah Berlin, « The Concept of Scientific History », dans Philosophical Analysis and History, op. cit., p. 40-51. Sur la « colligation » en général, voir les remarques de Mink, «The Autonomy », art. cit., p. 171-172. 


\section{Labyrinthe, $n^{\circ} 33$}

lambda. L'historien relie ensuite l'événement qu'il doit expliquer par différents « fils » qui partent dans toutes les directions; certains relient l'événement au contexte historique ambiant, d'autres à des éléments contextuels passés pour expliquer son « origine », d'autres encore à des éléments contextuels futurs, pour déterminer son « impact». Cette filiation s'arrête quand un fil se perd dans le contexte d'un autre événement, ou quand plusieurs fils convergent pour produire un nouveau contexte propice à de nouveaux événements. La motivation de l'historien n'est pas ici d'intégrer dans un même processus signifiant tous les événements ou tous les trends historiques observables, mais plutôt de les relier de manière qu'ils forment une chaîne explicative, provisoire et limitée à des phénomènes manifestement « significatifs ».

Il semble évident que l'approche contextualiste veuille ici concilier l'approche formiste, avec ses tendances à la dispersion, et l'approche organiciste, qui intègre à tout va. En fait, cette approche de la vérité (expliquée, vérifiée) manque d'ambition vis-à-vis et de l'historien et du lecteur; en même temps, en re-territorialisant le champ historique à l'aide de « bornes significatives », en distinguant des « périodes » et des « époques » distinctes les unes des autres, le contextualisme apporte une solution ambiguë au problème de la narration d'ensemble du processus historique. Le «flot» de l'histoire devient, chez les contextualistes, une succession de "vagues » (la métaphore se trouve d'ailleurs chez Burckhardt), plus ou moins « hautes » et conséquentes. L'opération qui consiste à relier par des fils différents événements pour suggérer des trends structurants offre bien la possibilité d'un récit évolutionniste du développement dans l'histoire. Toutefois, les stratégies explicatives tendent à privilégier la présentation synchronique de différentes séquences simultanées du processus historique, comme si l'on pouvait se faufiler à travers l'épaisseur historique en ignorant les à-côtés. Inévitablement, l'historien d'obédience contextualiste favorise le mode de représentation synchronique. S'il veut agréger les différentes périodes qu'il a étudiées en une vue d'ensemble du processus historique, il lui faut sortir du cadre méthodologique qui est le sien, et adopter soit le point de vue mécaniciste - des « lois intemporelles » s'appliquant alors à l'ensemble des données historiques; soit le point de vue organiciste - la synthèse étant alors opérée sous des auspices téléologiques. 
Ayant défini ces quatre approches, nous devons admettre qu'elles offrent également, chacune à leur façon, une explication plausible aux événements historiques. Force est de constater, toutefois, qu'elles n'ont pas joui de la même autorité parmi les historiens, en tout cas depuis la professionnalisation de l'histoire et sa transformation en discipline universitaire, au début du XIX ${ }^{\mathrm{e}}$ siècle. Deux d'entre elles, l'approche formiste et l'approche contextualiste, ont dominé le champ d'étude de manière écrasante, jusqu'à définir une sorte d'orthodoxie. Lorsque des tendances organicistes ou mécanicistes se sont fait jour chez des orfèvres de la narration comme Ranke et Tocqueville, elles ont été considérées comme de malencontreuses exceptions à la norme. Et lorsque, emportés par leur élan, certains penseurs ont poussé la logique organiciste (Hegel) et mécanicistes (Marx) jusqu'à en faire la clef de voûte de leur système d'explication de l'histoire, ce fut une raison suffisante - et disqualifiante - pour que leur œuvre tombât dans la catégorie, ô combien suspecte, de la philosophie de l'histoire.

Bref, pour les historiens professionnels, le choix s'est limité en fin de compte au formisme et au contextualisme; les modèles hétérodoxes, mécaniciste et organiciste, furent marginalisés à la fois par les historiens dominants et par leurs alliés au sein du camp philosophique, c'est-à-dire ceux qui n'avaient que dédain pour une " philosophie de l'histoire " entachée de mythologie, d'erreur ou d'idéologie. Ainsi l'ouvrage de Karl Popper intitulé Misère de l'historicisme constitue-t-il un réquisitoire passionné contre ces deux modèles d'explication de l'Histoire'.

Comment expliquer cette hostilité radicale des historiens professionnels? Ils ne peuvent invoquer des raisons épistémologiques. Après tout, les études historiques s'organisent selon une logique de profession. La préférence pour tel ou tel mode d'explication de l'histoire ne saurait, par conséquent, présenter un caractère d'universalité et de nécessité absolue.

Bien sûr, on a plaidé que l'histoire ne s'affranchirait des mythes, de la religion et de la métaphysique qu'à la condition d'exclure de son modus operandi les approches organiciste et mécaniciste. Certes, nous dit-on, cela ne suffirait pas à faire de l'histoire une science exacte; du moins éviterait-on les dangers du scientisme, cette manie de singer les méthodes des sciences pour asseoir une pseudo-autorité scientifique.

1. Karl H. Popper, The Poverty of Historicism, Londres, Routledge \& Paul, 1961, p. 5-55. 


\section{Labyrinthe, $n^{\circ} 33$}

En excluant toute autre approche que le formisme et le contextualisme, l'historiographie se garderait de toute sortie hors de l'empirie, et ne risquerait pas de s'aventurer dans les zones dangereuses de la philosophie de l'histoire à la Hegel ou à la Marx.

Mais, précisément, parce que l'histoire n'est pas une science exacte, cette hostilité à l'encontre des approches organiciste et mécaniciste relève tout au plus d'un préjugé de la part des historiens établis. Si nous arrivons à faire la preuve que ces deux approches peuvent compléter utilement notre compréhension des mondes naturels et sociaux, alors leur exclusion ne peut se justifier par des raisons strictement épistémologiques. L'attachement exclusif des historiens au formisme et au contextualisme traduit seulement leur refus de principe de tenter une opération de synthèse des données à la manière des historiens organicistes ou mécanicistes. Personne ne critique cette décision, qui ne fait que reconduire des opinions préétablies quant à la forme que doivent prendre les sciences humaines et sociales. Ces opinions relèvent donc d'une certaine éthique, et, pour tout dire, de choix idéologiques.

Pour l'extrême-gauche, en tout cas, c'est une affaire entendue: la préférence manifeste des historiens pour les schèmes contextualiste et formiste s'explique par des raisons idéologiques. Les intellectuels marxistes, par exemple, tiennent qu'il est dans l'intérêt des classes dominantes de rejeter les modes d'explication mécanicistes de l'histoire, dans la mesure où la mise au jour subversive des mécanismes de domination saperait les fondements de leur autorité et leurs privilèges. Dès lors, la seule conception de l'histoire autorisée se limite à rapporter les actions et les relations individuelles à un contexte immédiat; l'agencement narratif des faits aboutit tout au plus à de vagues généralisations, conformes aux préconceptions individualistes et hiérarchiques propres à la société « libérale-conservatrice».

Par contraste, les mêmes intellectuels marxistes, qui prétendent avoir découvert les « lois scientifiques de l'histoire », sont taxés d'idéologues par les historiens libéraux, pour qui on ne saurait mettre l'histoire au service d'un projet sulfureux de transformation de la société dans un sens révolutionnaire ou réactionnaire: un tel soupçon est de nature à décourager la tentation marxisante de chercher des « lois » expliquant la structure et les changements historiques, ou celle, propre aux idéalistes de type hégélien, de publier des ouvrages sur le sens universel de 
l'histoire. À noter sur ce point que les contextualistes, les formistes et les mécanicistes ont la même aversion pour les intentions « rétrogrades » et « obscurantistes » des amateurs de « principes innervant l'histoire».

En fait, toute description historique de la réalité a forcément une forte composante idéologique. L'histoire, comme on l'a dit, et quelles que soient ses prétentions en ce domaine, $n$ 'est pas une science; tout au plus une proto-science. Ainsi, parce que l'histoire $n$ 'est pas une science, mais est tout au plus une proto-science constituée d'éléments que l'on peut spécifiquement déterminer comme non-scientifiques, le fait même de prétendre avoir distingué quelque espèce de cohérence formelle dans les archives charrie avec soi des théories sur la nature du monde historique et du savoir historique même, et qui possèdent des implications idéologiques dans leur essai de compréhension du «présent », quelle que soit par ailleurs la manière de définir ce « présent ». Pour l'exprimer autrement, quand l'historien pose la distinction théorique entre passé et présent, et en même temps opère dans un continuum qui permet de rendre compte du passé dans le présent, il prend position sur la question du savoir, et de la forme qu'il doit prendre. Cette attitude détermine en retour le type de généralisations qu'il formule à propos du monde dans lequel nous vivons, la connaissance que nous pouvons en avoir, et nos aspirations légitimes à le transformer ou au contraire à le maintenir indéfiniment tel qu'il est.

\section{Explication par les motivations idéologiques}

La dimension « idéologique » d'une description historique reflète les dispositions éthiques de l'historien vis-à-vis de questions comme la nature de la connaissance historique et l'utilité de l'histoire pour comprendre le présent. Par « idéologie », j'entends donc l'ensemble des lois morales que se prescrit l'historien en vue de se positionner concrètement par rapport à la nécessité de changer le monde, ou au contraire de le conserver en l'état. À l'origine de ces prescriptions, on trouve des arguments du genre « la science prouve que... », ou « il faut être réaliste ». M'inspirant de Mannheim, dans son Idéologie et Utopie, je postule qu'il existe quatre 
grandes positions idéologiques : l'anarchisme, le conservatisme, le socialisme utopique [Radicals], et le libéralisme de gauche [liberalism $]^{1}$.

Il existe bien entendu d'autres positions « métapolitiques »; Mannheim cite la vision apocalyptique de certaines sectes religieuses du début de l'époque moderne, dont le projet est censé être révélé aux hommes par Dieu; la Réaction, qui voit un groupe d'individus, voire une classe entière, prôner la restauration du statu quo ante; ou encore le fascisme, qui repose sur l'autorité incontestée d'un leader charismatique. Toutefois, le degré d'autoritarisme qu'elles supposent est resté largement inconnu au

1. Je simplifie ici le système de Mannheim, qui comprend cinq grandes idéologies prenant appui sur autant de philosophies de l'Histoire, toutes apparues au XIX ${ }^{\mathrm{e}}$ siècle sous la forme de types-idéaux [au sens wébérien]. Mannheim dit cinq, et non quatre, car il distingue deux sortes de conservatisme, l'un « bureaucratique », l'autre « historiciste »-distinction dont je n'ai que faire ici, étant donné que le « bureaucrate » s'oppose en quelque sorte à toutes les idéologies prônant un changement dans ou de la société. Ce qui m'intéresse est le travail des intellectuels qui veulent rompre avec le statu quo au nom de conceptions philosophiques du processus historique. Pour autant que je sache, aucun historien ou philosophe n'a jamais cherché à promouvoir le « conservatisme bureaucratique ». Toutefois, dès lors que le conservatisme tel que je le définis vise à défendre non pas un passé idéalisé, mais bien l'ordre social existant, le « conservatisme historiciste » tel que se le représente Mannheim constitue le refuge naturel des bureaucrates conservateurs. Voir Karl Mannheim, Ideology and Utopia, New York, Brace, Harcourt \& C $C^{\circ}$, 1946, p. 104 sqq.; et « Conservative Thought », dans Paul Kecskemeti (dir.), Essays on Sociology and Social Psychology, New York, Oxford University Press, 1953, p. 174-153. Pour le $\mathrm{XX}^{\mathrm{e}}$ siècle, Mannheim nomme une sixième option idéologique, le fascisme. Je n'ai pas utilisé cette catégorie, qui serait anachronique pour les penseurs du XIX ${ }^{\mathrm{e}}$ siècle - même si l'idée que Mannheim se fait de l'approche « apocalyptique » de la politique sous le nom d'anarchisme n'est en un sens pas très éloignée du « fascisme ». Par ailleurs, dans son essai intitulé La Mentalité utopiste, Mannheim distinguait quatre formes d'utopie comme autant d'étapes dans l'évolution de la conscience politique moderne: la croyance millénariste portée par les Anabaptistes au XVI ${ }^{e}$ siècle; l'humanitarisme des Lumières; l'idéal bourgeois-conservateur; enfin l'utopie communiste. L'anarchisme au XIX ${ }^{\mathrm{e}}$ siècle, le fascisme $\mathrm{au} \mathrm{XX}^{\mathrm{e}}$ siècle ne sont jamais que les succédanés laïques de la tradition millénariste et apocalyptique. Ce qui fait le caractère unique de l'anarchisme c'est qu'à la différence du millénarisme et du fascisme, il prend ses responsabilités au plan cognitif, c'est-à-dire qu'il cherche à justifier rationnellement une posture irrationnelle. Par ailleurs, je note qu'il s'est répandu au XIX ${ }^{\mathrm{e}}$ siècle dans le sillage du romantisme, qui a enfanté le fascisme au Xx ${ }^{\mathrm{e}}$ siècle. Je suis sur ce point en désaccord avec Mannheim, pour qui romantisme et conservatisme vont de pair, sous prétexte que les premiers auteurs romantiques furent contemporains de la formulation de l'idéal conservateur. En fait, la philosophie de l'Histoire prophétisée par les mages romantiques n'est pas compatible avec l'idéal d'une société intégratrice et ordonnée au terme d'un long processus historique, qui fait des conservateurs les chantres du statu quo social. Au contraire, l'exaltation sans précédent de l'individualisme inspire aux romantiques le désir d'une parfaite anarchie. Certes, on trouve une tentation égoïste au sein de la famille conservatrice, chez certains penseurs originaux; toutefois, le vrai conservateur ne peut recourir à l'argument individualiste que pour des raisons stratégiques, afin de défendre les intérêts de groupes privilégiés, contre les menées séditieuses des socialistes, des libéraux, et même des réactionnaires. La conception anarchisante du monde lui est tout aussi odieuse que les programmes socialistes. Anarchistes et socialistes rêvent d'un monde en harmonie; pour lui, cette unité organique est déjà réalisée. 
$\mathrm{XIX}^{\mathrm{e}}$ siècle. En outre, bien que des porte-parole de ces doctrines consentent parfois à polémiquer avec leurs opposants, ils n'ont aucun souci de fonder leur approche cognitive du monde sur des bases scientifiques ou rationnelles. Dès lors, bien qu'ils proposent certaines théories de la société et de l'histoire, on ne saurait tout à fait les prendre au sérieux, ni les faire participer au débat critique, puisque leur approche ne répond pas aux standards minimaux de rigueur et de cohérence. Au contraire, les quatre grandes positions idéologiques identifiées par Mannheim revendiquent leur ancrage dans « la raison », ou dans « la science ». Ce point de départ implique une volonté de dialoguer avec d'autres systèmes de pensée qui se soumettent au même principe d'autorité épistémologique et à la même éthique de la responsabilité scientifique, ce qui forme un cadre de discussion que rejettent les partisans de l'autoritarisme. Même si les points de vue sur la société diffèrent, chacun est prêt à tenir compte dans sa propre recherche des données mises au jour par l'adversaire idéologique. Bref, l'anarchisme, le conservatisme, le socialisme utopique et le libéralisme jouent le jeu de la responsabilité cognitive, à la différence des partisans de systèmes autoritaires ${ }^{1}$.

Je crois utile ici de préciser que les termes « anarchiste », « conservateur », « socialiste utopique » et « libéral de gauche » désignent des préférences idéologiques générales plus que des partis politiques précis. Selon son affiliation, l'historien estimera plus ou moins possible, et souhaitable, que la société fasse l'objet d'une étude scientifique; il croira plus ou moins que l'on peut tirer des « leçons » politiques de l'enseignement des sciences humaines; il sera plus ou moins favorable au statu quo, ou au changement social, et favorisera telles ou telles voies politiques; enfin, il regardera plutôt vers le passé, le présent, ou l'avenir, comme

\footnotetext{
1. J'emprunte la notion de responsabilité cognitive [cognitive responsibility] à Pepper, qui distingue entre les philosophes selon qu'ils acceptent ou non le principe de rationalité pour justifier leurs hypothèses sur la marche du monde. Dans la seconde catégorie, on trouve les penseurs mystiques, animistes, et sceptiques: tous sont amenés, tôt ou tard, à faire appel soit aux notions d'autorité divine (révélée ou non), soit à des conventions, pour justifier leurs arguments. Les rares fois où ils font appel à la raison pour justifier leur posture fondamentalement irrationnelle, il s'agit de dénoncer « l'hyper-rationalisme » de leurs contradicteurs. Leurs doctrines sont par essence indéfendables sur un mode rationnel, dès lors que chez le mystique, l'animiste, et le sceptique, la Raison n'a jamais le dernier mot. Voir Pepper, World Hypotheses, op. cit., p. 115-37. Des attitudes équivalentes au plan politique seraient celles de la noblesse féodale liée par la tradition; du réactionnaire, qui dénie toute valeur au présent comme au futur; et du fasciste nihiliste, qui ne reconnaît ni la raison, ni l'idéal de cohérence interne dans le débat avec ses opposants.
} 


\section{Labyrinthe, $n^{\circ} 33$}

le creuset de la société « idéale ». Je dois aussi insister sur le fait que le choix de telle ou telle forme narrative ou le recours à tel ou tel paradigme historique ne vont pas forcément de pair avec telle ou telle revendication d'appartenance à une idéologie. C'est en fait plutôt le contraire qui se produit: c'est la forme narrative choisie par l'historien qui le rattache à une idéologie. Au risque de me répéter, j'affirme donc ceci: de même que chaque idéologie est porteuse a priori d'un certain rapport à l'histoire, de même chaque façon de raconter l'histoire a forcément certaines implications idéologiques.

Passons maintenant à la caractérisation des différentes idéologies, en prenant pour critère leur position vis-à-vis du changement social. Les quatre grandes idéologies s'accordent à penser que le changement est inévitable à terme; mais elles diffèrent sur la question de savoir si le changement est souhaitable, et sur le rythme de la réforme. Les conservateurs sont bien sûr les plus réticents face à la perspective de rompre avec le statu quo; les libéraux de gauche, les anarchistes et bien sûr les socialistes utopiques sont plus favorables au changement social; ils sont en outre relativement optimistes quant à la possibilité de transformer rapidement la société. Comme le faisait remarquer Mannheim, les conservateurs favorisent l'analogie entre le mouvement des sociétés et la croissance lente des plantes, tandis que les libéraux de gauche (en tout cas ceux du XIX ${ }^{\mathrm{e}}$ siècle) utilisent plus volontiers la métaphore des mécanismes sociaux, qu'il est possible d' « ajuster » et de « réguler» différemment. Mais libéraux et conservateurs se retrouvent sur un point: ils considèrent que les structures fondamentales de la société sont saines et solides. S'il est inévitable que certains changements se produisent, il vaut mieux qu'il s'agisse de changements partiels, plutôt que d'une altération complète des rapports institutionnels, sans parler d'un bouleversement de l'ordre existant. Les socialistes utopiques et les anarchistes, en revanche, croient dans la nécessité de transformations structurelles, les premiers afin de reconstruire la société sur des bases nouvelles, les seconds avec l'objectif d'abolir purement et simplement la société, et de lui substituer une communauté universelle du genre humain.

Pour ce qui est du rythme du changement, les conservateurs tiennent à l'idée d'une évolution « naturelle » des choses, tandis que les libéraux de gauche favorisent le rythme social de la réforme, celui des débats parlementaires, de l'éducation progressive des masses, et du processus 
électoral, le tout dans le respect de l'État de droit. À l'inverse, les socialistes utopiques et les anarchistes ne reculent pas devant la nécessité d'un changement brutal et cataclysmique de la société, même si les premiers sont plus conscients que les seconds des difficultés pratiques pour opérer ce changement; ils sont plus sensibles, en particulier, à l'inertie conservatrice des institutions, et croient par conséquent que certaines conditions préalables sont requises. Du coup, ils acceptent de différer la révolution.

Cela nous conduit à la question du rapport à la temporalité historique des différentes idéologies. Si l'on en croit Mannheim, les conservateurs voient dans l'évolution historique la lente et patiente édification de structures institutionnelles qui, à un moment donné, représentent la meilleure des sociétés possibles; pour tout esprit raisonnable, sa conservation est par conséquent la seule « utopie » légitime. Les libéraux de gauche, en revanche, imaginent que cette structure fera l'objet d'améliorations dans le futur; toutefois, ce futur est présenté comme lointain, de façon à décourager toute velléité d'opérer le changement de manière précipitée, et par des moyens radicaux. Les socialistes utopiques, quant à eux, considèrent que les conditions sont presque rassemblées pour un changement de société, ce qui les motive pour préparer la révolution. Enfin, les anarchistes sont portés à la nostalgie d'un " état de nature » idéal: « il y a très longtemps » (on n'est pas plus précis), les hommes vivaient dans l'innocence; puis est venue la Chute, sous la forme de l'existence sociale. Il est pourtant permis de renouer à tout moment avec cette utopie intemporelle: il suffit pour cela que les êtres humains décident à nouveau de ne pas se laisser corrompre et de briser le carcan de la société; cet acte de la volonté transcenderait la croyance, imposée par l'élite, dans la légitimité des institutions sociales.

La relation particulière que chaque idéologie entretient avec la dimension temporelle de l'utopie conduit Mannheim à poser que les approches de la société sont soit congruentes, soit transcendantes. Le conservatisme est par excellence une idéologie congruente (insistant sur la nécessite de faire avec), ce qui le rapproche jusqu'à un certain point du libéralisme. L'anarchisme au contraire se réclame de la transcendance (il faut aller au-delà du possible), ce qui le rapproche jusqu'à un certain point du socialisme révolutionnaire. Mais à y regarder de plus près, chacune de ces quatre idéologies est un composé des deux attitudes, et les différences affectent plus le discours que le contenu. Toutes prennent quoi qu'il en 
soit les changements sociaux au sérieux, d'où l'intérêt porté à l'histoire en tant qu'elle fournit la justification historique des programmes politiques; d'où également la volonté d'avoir conséquemment un débat responsable portant sur le rythme et sur les modalités du changement.

C'est bien plutôt le jugement porté sur l'élite en place qui décide des conceptions de l'évolution historique, et de la connaissance de l'histoire. Selon Mannheim, il existe plusieurs façons d'envisager le développement humain dans l'histoire. Ce qui constitue un progrès pour l'un n'est que symptôme d'une décadence pour l'autre; pour ce qui est du présent, il est vu comme un âge d'or ou comme un âge de fer, en fonction du degré d'aliénation de l'observateur. En même temps, chaque tendance idéologique favorise un certain paradigme d'explication des faits historiques. La forme que prend cette explication reflète une conception de « la scientificité de l'histoire » propre à chaque idéologie. C'est ainsi que les socialistes révolutionnaires et les libéraux croient également dans la possibilité d'étudier l'histoire en suivant une méthode rationnelle et scientifique, même s'ils ne cherchent pas la même chose: les premiers veulent définir des lois régissant les structures et les processus historiques, tandis que les seconds ne font en général que repérer les trends de l'évolution générale des sociétés. De leur côté, conservateurs et anarchistes restent fidèles aux conceptions très positivistes du XIX ${ }^{\mathrm{e}}$ siècle, selon lesquelles il est possible de donner au « sens de l'histoire » la forme d'un grand schéma conceptuel, certes compatible avec les exigences du débat rationnel, mais qui repose avant tout sur l'intuition d'un certain nombre de phénomènes. L'anarchiste tend à montrer une empathie toute romantique dans son récit des faits historiques; le conservateur veut fusionner les différentes intuitions que lui inspirent les faits dans un vaste système organiciste qui rende compte de l'ensemble du processus historique.

De mon point de vue, le seul terrain sur lequel on puisse arbitrer entre différentes conceptions du processus historique et du savoir historique est un terrain idéologique. En effet, dès lors que ces conceptions irréconciliables ont une origine éthique, on ne saurait juger de leur valeur cognitive qu'en faisant le choix éthique d'une position épistémologique. Qualifier l'une ou l'autre de ces conceptions du savoir historique de « plus réaliste » que les autres n'a pas de sens pour moi, puisque le différend idéologique porte précisément sur les critères du réalisme. Je me refuse également à décerner les qualificatifs de "scientifique » ou 
« non scientifique » à tel ou tel type d'historien tant que la question n'est pas réglée de savoir ce que devrait être la science historique ou sociale par rapport aux autres sciences.

Naturellement, il en allait différemment au XIX ${ }^{\mathrm{e}}$ siècle, où la plupart auraient répondu: «C'est l'approche mécaniciste qui est la plus scientifique. » Et pourtant, les sociologues continuaient de débattre de la scientificité d'une telle approche de la société et de l'histoire. La floraison de travaux théoriques d'inspiration formiste, organiciste et contextualiste prouvait à elle seule la persistance d'opinions divergentes quant au bienfondé du mécanicisme.

Je ne me soucie donc pas d'établir un palmarès des différentes conceptions de l'histoire produites au XIX ${ }^{\mathrm{e}}$ siècle en vertu de leur degré de réalisme ou de scientificité. Je ne souhaite pas davantage les analyser en tant que déclinaisons d'idéologies. Ce qui m'intéresse, c'est la prise en compte des considérations idéologiques dans l'évaluation des théories et modes d'écriture en tant qu'elles participent des efforts de l'historien dans ce domaine. D'un autre côté, je voudrais démontrer que même lorsque des historiens et des philosophes de l'histoire ont un objectif qui n'est manifestement pas politique, comme c'est le cas pour Burckhardt ou Nietzsche, leurs travaux ont à tout le moins certaines résonances, et toujours de profondes implications idéologiques.

J'estime que l'on entre dans le domaine de l'éthique quand la combinaison d'une certaine perception esthétique des choses, qui va de pair avec un mode de narration, et d'une certaine perception des enjeux cognitifs, qui va de pair avec un type d'argument, résulte en prescriptions idéologiques, sur la base d'éléments a priori purement descriptifs ou analytiques. Un historien peut expliquer des événements par une ou plusieurs loi(s) gouvernant l'histoire en ayant recours au mode narratif de la tragédie. À moins que la tragédie ne consiste précisément dans la découverte qu'il existe de telles lois. Dans les deux cas, les implications éthiques d'un argument historiographique résultent de la combinaison idoine d'un certain type de structure narrative et d'un certain type d'explication présentée comme « scientifique » ou « réaliste », combinaison à laquelle se prêtent les événements étudiés.

Par exemple, une série d'événements « tragiques », c'est-à-dire relatés comme une tragédie, peut trouver une explication d'allure « scientifique » dans l'invocation de strictes lois de détermination des causes et des 


\section{Labyrinthe, $n^{\circ} 33$}

effets, impliquant un certain fatalisme: les hommes ne peuvent échapper à leur destinée historique. La tragédie peut, au contraire, résider dans la prise de conscience qu'il existe des lois, mais la résistance que leur opposent les hommes, ou du moins leur capacité à infléchir ces lois, témoigne de leur capacité à faire l'histoire. On a tôt fait de rapporter ces deux alternatives à la Réaction (on pense à Spengler), ou à l'utopie révolutionnaire - et le nom de Karl Marx vient alors à l'esprit. De fait, même quand l'historien n'annonce pas clairement la couleur, on trouve toujours une certaine tonalité, un mode majeur, dans la mise en musique du « drame » des événements. Chaque historien use différemment de la même stratégie d'explication historique: chez Spengler, coexistent des lois mécaniques, une tonalité générale tragique, mais aussi une certaine faculté d'adaptation à la complexité des phénomènes sociaux. Dans l'œuvre de Marx, au contraire, des lois tout aussi mécaniques révèlent une vision tragique, mais aussi militante et héroïque de l'histoire. La même différence existe entre Euripide et Sophocle, ou, pour s'en tenir à un seul auteur, Shakespeare, entre Le Roi Lear et Hamlet.

Le lien logique entre forme narrative, intention idéologique et schéma explicatif est évident quand, par exemple, Ranke choisit la forme de la comédie, pour mettre en scène le thème central chez lui de la réconciliation, et intègre tous les événements dans un système de type organiciste. Il ne visait pas à mettre au jour des « lois», mais à décortiquer les « grandes idées » qu'il considérait comme les véritables agents de l'histoire. Je soutiens qu'il existe un lien épistémologique très fort entre son approche théorique des faits, sa perception esthétique du champ historique et le choix narratif d'une certaine forme omniprésente dans ses écrits, la comédie. Les implications idéologiques de cette combinaison sont typiques d'un historien conservateur comme Ranke; pour lui, tout dans l'histoire résulte en harmonie, ce qui est également le cas dans la comédie, genre conservateur. Comme la fin de la comédie, le lecteur de son œuvre historique contemple satisfait la résolution des oppositions $a$ priori tragiques, chaque « idée » (institution, valeur...) trouvant sa place et son sens dans un système organique dont chacun finit par s'accommoder. L'humeur dominante est badine et optimiste, et l'état d'esprit franchement conservateur - puisque, face à une telle construction historiographique, on ne saurait conclure autrement, en étant « réaliste », que l'on vit dans 
« le meilleur des mondes possibles » historiquement parlant, c'est-à-dire au regard des processus historiques mis à jour par Ranke.

Burckhardt offre un autre type de combinaison. D'une part, il tentait d'expliquer les événements en contexte, en reconnaissant la singularité des différents acteurs ou paramètres de l'espace historique. D'autre part, il ne croyait pas à la possibilité de dériver des lois scientifiques de l'étude de l'histoire, et évitait de réduire l'analyse des faits à une typologie. L'histoire lui apparaît comme le théâtre d'actions plus ou moins brillantes, qu'il peut représenter de manière plus ou moins impressionniste. Pour cette raison, d'aucuns estiment que son livre le plus connu, La Civilisation de la Renaissance, ne contient aucune « histoire » et ne suit aucune ligne narrative. Cela n'a rien d'étonnant, puisque Burckhardt privilégie la forme satirique, au sens étymologique ${ }^{1}$; l'ironie burckhardtienne n'est pas compatible avec le type de cohérence formelle que l'on attend de la romance, de la comédie, ou de la tragédie. Son idéologie, faite d'hostilité aux « idéologies », et son attitude sceptique vis-à-vis de la connaissance historique se présentent comme une alternative aux philosophies de l'histoire théorisées par Marx, Hegel et Ranke, trois penseurs pour lesquels il n'avait que mépris. Elles trouvent une forme idoine sur le plan esthétique, la satire.

Toutefois, la satire peut avoir des implications idéologiques différentes, selon que l'auteur est un libéral optimiste, ou un conservateur fataliste. Prenons l'exemple de Burckhardt: entre les différents événements - du moins ceux que leur brillance rend dignes d'être relatés -, il refuse de voir d'autres liens que ceux de leur contemporanéité. Cette position et le scepticisme ironique de son style découragent par avance toute tentative non conservatrice d'utiliser l'histoire dans le présent. Sa vision très pessimiste de l'avenir a au contraire pour effet d'encourager chez ses lecteurs un réflexe de « sauve qui peut », l'idée qu' ' il ne reste plus qu'à sauver les meubles ». Certes, une telle mentalité est de nature à servir aussi bien des causes libérales que des causes conservatrices ${ }^{2}$. En revanche, on n'imagine guère qu'un programme révolutionnaire puisse prendre appui sur l'historiographie burckhardtienne, dont les implications sont, au mieux, conservatrices, au pire, réactionnaires.

1. Note du traducteur: Satura désigne à l'origine une sorte de salade faite de raisins secs, de polenta et de pignons, d'où l'idée de genre littéraire composite, ou mélange.

2. En français dans le texte. $[\mathrm{NdT}]$ 


\section{Labyrinthe, $n^{\circ} 33$}

\section{La question des styles dans l'historiographie}

Ayant distingué les trois niveaux historiographiques d'explication, je dois à présent m'attacher à la question des styles. Par « styles », j'entends les manières de combiner différents types de narrations, d'argumentations et d'idéologies sous-jacentes. Toutes les combinaisons ne sont pas possibles: par exemple, le genre de la comédie « ne marche pas » avec l'approche mécaniciste; l'idéologie socialiste révolutionnaire ne s'accommode pas de la satire, etc. Pour l'historien qui cherche à convaincre à chaque étape de son récit, il existe, pour ainsi dire, des affinités électives ou des homologies structurales entre les modes d'écriture, les types d'argumentation et les postures idéologiques. Ces affinités peuvent être visualisées de la façon suivante:

$\begin{array}{lll}\text { Intrigue } & \text { Argument } & \text { Implication idéologique } \\ \text { Romantique } & \text { Formiste } & \text { Anarchiste } \\ \text { Tragique } & \text { Mécaniciste } & \text { Socialiste révolutionnaire } \\ \text { Comique } & \text { Organiciste } & \text { Conservateur } \\ \text { Satirique } & \text { Contextualiste } & \text { Libéral de gauche }\end{array}$

Qu'il y ait certaines affinités ne veut pas dire que les historiens optent nécessairement pour les combinaisons attendues. Au contraire, ce qui fait le chef-d'œuvre historique, c'est la tension qui résulte de noces « contrenature » entre un certain mode narratif, un certain type de démonstration, et un certain propos idéologique... Bien qu'ils semblent a priori s'exclure mutuellement. Michelet tente de marier le drame romantique, la logique formiste et une idéologie ouvertement libérale; Burckhardt peut mettre la satire au service d'arguments contextualistes et d'une pensée ouvertement conservatrice, pour ne pas dire réactionnaire; Hegel fait jouer l'argument organiciste à la fois au niveau micro-historique et au niveau macro-historique, avec pour effet qu'en changeant d'échelle, le lecteur passe de la tragédie à la comédie - et en tire des conclusions soit révolutionnaires soit conservatrices. Ces tensions dialectiques ne prennent leur sens qu'en fonction d'une conception totalisante de l'histoire, qui procure à l'œuvre son unité et son unicité. De mon point de vue, ce qui fait qu'une œuvre est cohérente et idiosyncrasique, c'est sa poétique et ses fondements linguistiques. 
Avant même que l'historien ne "plaque » son appareil conceptuel et rhétorique sur les données qu'il a collectées au sein du champ historique, il doit se faire une image pré-conceptuelle de ce dernier. Il s'agit là d'un acte poétique, d'un découpage linguistique du réel: avant d'être interprété, le domaine d'étude doit être construit et « peuplé » de figures bien identifiables et hiérarchisées, qu'il s'agisse d'ordres, de classes, de genres ou d'espèces, dont les relations changeantes constituent autant de " problèmes » à résoudre au cours de la narration et de l'argumentation.

Autrement dit, devant le champ historique, l'historien se trouve dans une situation qui ressemble beaucoup à celle du grammairien confronté à un nouveau langage. Son premier souci est de distinguer les éléments lexicaux, grammaticaux et syntaxiques qui structurent son champ d'étude. Ce n'est que dans un second temps qu'il peut se lancer dans l'interprétation de telle configuration ou de telle modification des rapports de force à l'intérieur du champ. Bref, son problème est de construire un protocole linguistique complet, qui lui permette de définir de manière détaillée les caractéristiques lexicales, grammaticales, syntaxiques, et sémantiques de son champ d'étude - et cela d'une manière qui n'appartient qu'à lui, et non pas en fonction d'une terminologie pré-attribuée par les documents. Ce faisant, il prépare le terrain pour sa narration et son argumentation. Ce protocole relève d'un certain mode tropologique.

Les récits d'événements historiques sont censés être des modèles, ou des icônes, qui rendent compte par écrit de segments spécifiques du processus historique. Mais s'il est nécessaire de disposer de " modèles ", c'est que les archives seules ne permettent pas de se faire une image univoque et structurée des événements. C'est pourquoi, pour une présentation effective de «ce qui s'est réellement passé », l'historien doit commencer par se représenter l'ensemble des événements rapportés dans le document d'archives comme un tout susceptible d'enrichir notre connaissance du passé.

On peut dire que cet acte pré-figuratif est poétique dans la mesure où, pour l'historien, il précède toute conscience cognitive, ou toute conscience critique. Poétique, il l'est aussi au sens étymologique de poeiein, créer: ce qui est en germe est une création verbale, seul moyen pour l'historien d'offrir sa représentation et son explication de « ce qui s'est réellement passé ». Le domaine de l'histoire qui devient image mentale, les concepts qui vont être utilisés pour identifier les objets et caractériser leurs rela- 
tions à l'intérieur du champ d'étude, sont prédéfinis. Bref, tout se passe comme si l'historien-poète, avant même de commencer l'analyse, créait son objet, et faisait le choix de ses stratégies d'explication à venir. Ce choix n'est toutefois pas infini. Il dispose en fait de quatre stratégies, qui correspondent aux quatre principaux tropes de la rhétorique: ironie, métonymie (« le contenant pour le contenu »), métaphore, et synecdoque ( «la partie pour le tout »). L'écriture de l'histoire opère à intérieur des limites du langage poétique ${ }^{1}$.

1. Les deux grands défenseurs de la conception tropologique du discours non scientifique (qu'il soit mythique, artistique, ou onirique) sont deux structuralistes, Roman Jakobson et Claude Lévi-Strauss. Le premier a recours à la dyade métaphore/métonymie comme fondement de son analyse des logiques nominales dans les cultures primitives: c'est pour lui la clef de compréhension des mythes. Voir $L a$ Pensée sauvage, op. cit., chapitres 7 et 8 , et, pour un résumé de la méthode lévi-straussienne, Edmund Leach, Claude Lévi-Strauss, New York, Viking Press, 1970, p. 47 sqq. Jakobson utilise la même dyade pour fonder sa théorie linguistique de la littérature. Voir son brillant essai «Linguistics and Poetics » dans Style in language, Thomas A. Sebeok (dir.), New York et Londres, 1960, ainsi que le fameux chapitre 5 de l'ouvrage de Roman Jakobson et Morris Halle, Fundamentals of Language, 's-Gravenhage, Mouton, 1956, intitulé « The Metaphoric and Metonymic Poles », repris dans Hazard Adams (dir.), Critical Theory since Plato, New York, Harcourt Brace, Jovanovich, p. 1113-1116. Pour une application au domaine des rêves, voir Jacques Lacan, « Le séminaire sur la lettre volée, L'instance de la lettre dans l'inconscient », dans Écrits, Paris, Le Seuil, 1966. Lévi-Strauss, Jakobson et Lacan conçoivent tous trois la métaphore et la métonymie comme les « pôles » de l'acte de communication, selon que le speech act s'exprime selon un axe continu (verbal) ou discontinu (nominal). Dans la théorie stylistique de Jakobson, la synecdoque et l'ironie ne sont que des sous-catégories de la métonymie, qu'il considère comme le trope fondamental de la prose « réaliste ». Voir « Linguistics and Poetics », p. 375. Il convient toutefois de rappeler que le premier à avoir proposé une analyse de l'histoire du réalisme selon la grille métonymique fut Stephen Ullmann dans son livre Style in the French Novel, Cambridge, Cambridge University Press, 1967. Indéniablement, l'approche dyadique [Note du traducteur: la dyade repose sur la distinction entre la signification linguistique et le « contenu » de l'énoncé] a donné des résultats remarquables dans le domaine strictement linguistique. Je crois cependant qu'elle a ses limites pour l'analyse stylistique. Je suis donc tenté de reprendre le système quaternaire inventé à la Renaissance pour nommer les différentes conventions stylistiques. C'est bien en effet d'enjeu stylistique qu'il s'agit, comme Benveniste l'a suggéré dans son essai perspicace sur la théorie freudienne du langage : «C'est dans le style, plutôt que dans le langage, que nous verrions un terme de comparaison avec les propriétés que Freud a décelées comme signalétiques du « langage » onirique... L'inconscient use d'une véritable « rhétorique », qui, comme le style, a ses « figures », et le vieux catalogue des tropes fournirait un inventaire approprié aux deux registres de l'expression [le symbolique et le sensible]. » Emile Benveniste, « Remarques sur la fonction du langage dans la découverte freudienne, dans Problèmes de linguistique générale, Paris, Gallimard, 1966, vol I, p. 86. Dans cet essai, Benveniste abolit les distinctions traditionnelles entre prose et poésie, langage des rêves et langage de la conscience à l'état de veille, entre les pôles métaphorique et métonymique. Cela est cohérent avec ma propre objection sur le fait que les similarités entre les représentations poétique et discursive de la réalité sont aussi importantes que leurs différences. Car il en va des fictions « réalistes » comme il en va des rêves: « la nature du contenu fera apparaître toutes les variétés de la métaphore, car c'est d'une conversion métaphorique que les symboles de l'inconscient tirent leur sens et leur difficulté à la fois. Ils emploient aussi ce que la vieille rhétorique appelle la métonymie (contenant pour le contenu) et la synecdoque (partie pour le tout), et si la « syntaxe » 


\section{Poétiques de l'histoire}

des enchaînements symboliques évoque un procédé de style entre tous, c'est l'ellipse », Ibidem, p. 87. Il convient, lorsqu'on passe d'une analyse linguistique à une analyse stylistique des discours littéraires qualifiés de « réalistes », de ne pas confondre - comme on le fait trop souvent - tropes et figures de style d'un côté, tropes et schémas rhétoriques de l'autre. De ce point de vue, certains rhétoriciens subtils $\mathrm{du} \mathrm{XVl} \mathrm{l}^{\mathrm{e}}$ siècle, tout en distinguant clairement les quatre tropes, avaient une conception du fonctionnement stylistique beaucoup moins rigide et compartimentée que les linguistes contemporains. Tout en gardant la distinction fondamentale entre métaphore et métonymie, ils en vinrent ainsi à considérer la synecdoque comme une sorte de métaphore, et l'ironie comme une sorte de métonymie, ce qui leur permit de se concentrer sur les différences stylistiques les plus importances, celles qui donnent la mesure du degré de synthèse par réduction, ou au contraire de dispersion par création de sous-ensembles. Dans sa Science Nouvelle $(1725,1740)$, Giambattista Vico utilisait lui aussi le système tropologique quaternaire, pour différencier les étapes de la conscience à travers lesquelles l'humanité était passée, du primitivisme à la civilisation. Au lieu de voir une opposition entre la conscience poétique (mythique) et la conscience prosaïque (scientifique), Vico, donc, percevait une continuité. Voir Thomas G. Bergin et Max H. Fisch (trad.), The New Science of Giambattista Vico, Ithaca, New York., Cornell University Press, 1968, vol. 2, p. 129 sqq. Sur les théories de la rhétorique à la Renaissance, et pour un catalogue des modes de discours et des figures de style, voir Lee A. Sonnino, A Handbook to Sixteenth Century Rhetoric, Londres, Routledge \& Paul, 1968, p. 10-14 et 243-246. Mais que signifie la distinction toute conventionnelle entre schéma rhétorique et figure stylistique? D'un côté, nous disposons d'un ordre du langage (lexis) ou de la pensée (dianoia) qui n'admet ni « saut logique » ni possibilité de substitution; de l'autre, la figure de style consiste précisément à opérer cette substitution irrationnelle, ou au moins inattendue; soit, par exemple, le lexème « une passion froide ", quand on s'attendrait à trouver « passion brûlante ». Est-ce pour autant une formulation irrationnelle? Ne peut-on pas dire de toute figure de discours qu'elle est rationnelle, dès lors qu'elle produit l'effet voulu par le locuteur? Et l'usage créatif du langage ne consiste-t-il pas à accepter et même à rechercher des formulations qui s'écartent de la norme, au lieu de livrer à la conscience, et sur un plateau, ce qu'elle s'attend à lire, à penser, ou à entendre? Ce qui vaut pour la prose la plus réaliste comme pour la poésie la plus « romantique ». Les terminologies systématiques, comme celles utilisées pour l'analyse des données en physique, visent à éliminer du langage toutes les figures de style, afin que nul objet imprévisible et/ou innommable ne surgisse en cours de route. Par exemple, on se met d'accord sur un mode de calcul avant d'envisager le système de Newton, de sorte que le discours sur l'objet d'étude (et non l'objet lui-même) devient du coup entièrement schématique. Certes, même en science physique, par exemple lorsqu'ils passent d'une théorie de l'univers à l'autre, certains chercheurs utilisent la métonymie, et progressent par sauts logiques, en faisant du coup intervenir l'irrationnel dans leur démonstration. Toutefois, ces physiciens « créatifs » sont forcés de reformuler ces énoncés dans un langage qui leur permette de communiquer avec d'autres physiciens allergiques aux figures de style. L'obstacle fondamental, pour qui veut représenter de manière « réaliste » et « objective » des pans de l'expérience humaine pour lesquels il ne peut exister un tel carcan mathématique, c'est de faire coïncider la mise en mots avec le schéma de pensée jugé vrai envers la réalité. Or, quand il n'existe aucun consensus sur ce en quoi consiste le domaine d'expérience considéré, ni sur ce que serait sa vraie nature; ou, lorsque tout un chacun redéfinit de but en blanc certains phénomènes comme « la révolution », en faisant fi des conventions établies, alors la notion de « ce qu'on est en droit d'attendre » n'a plus aucun sens. Par conséquent, on ne saurait penser ni l'objet d'étude, ni son mode de représentation verbale, indépendamment des figures de style utilisées. Il est impératif, dans l'analyse de tout discours prétendument « réaliste », de déterminer le registre poétique dominant. C'est la seule façon d'accéder au niveau de conscience pré-réflexif où se trouve l'auteur quand il constitue le monde comme lieu de son expérience. À l'aide des quatre principaux tropes, les master tropes chers à Kenneth Burke, on peut mettre au jour ce qui est plus ou moins recouvert au sein de tout discours, «poétique » comme «prosaïque », sur la réalité: des styles de pensée. 


\section{Labyrinthe, $n^{\circ} 33$}

\section{La théorie des tropes}

Les tropes sont particulièrement utiles pour comprendre les opérations de saisie pré-figurative de pans entiers de l'expérience humaine, ceux qui résistent à la description du réel dans une langue prosaïque, objective et univoque.

Dans la métaphore (du grec «transfert»), les phénomènes peuvent êtres caractérisés en termes de similarité, d'analogie ou au contraire de différence, avec d'autres phénomènes: nous prendrons comme exemple « Mon amour, ma rose ». La métonymie (du grec « changement de nom »), elle, consiste à remplacer, dans le cours d'une phrase, le nom de la partie d'une chose par celui du tout, par exemple lorsqu'on emploie « voiles » pour «navires ». Avec la synecdoque, trope que certains théoriciens regardent comme une forme particulière de métonymie, même si nous verrons que les choses sont plus compliquées, le nom de la partie sert à symboliser une qualité présumée inhérente à la totalité; ainsi dans l'expression «il a le cœur sur la main ». Enfin, l'ironie est une forme d'expression qui consiste à dire figurativement l'inverse de ce que l'on déclare littéralement, tout en s'efforçant de laisser entendre la distance qui existe entre ce que l'on dit et ce que l'on pense réellement. Des formes particulières d'ironie sont la catachrèse, raccourci de langage a priori absurde (« un discours aveugle»), ou l'oxymore, qui met côte à côte deux mots ayant des sens opposés, et aboutissant à une image paradoxale, comme dans « une passion froide. »

L'ironie, la métonymie et la synecdoque sont en un sens des sortes de métaphores, mais au plan littéral chacune produit des effets différents, de réduction ou d'intégration, et contribue ainsi, au plan figuré, à faire voir

\footnotetext{
Voir Burke, Grammar, op. cit., appendice D, p. 503-517; et Paul Henle (dir.), Language, Thought, and Culture, Ann Arbor, Michigan, 1966, p. 173-195. La littérature sur les tropes est proliférante, et en proie à des dissensus congénitaux. Une analyse des problèmes rencontrés dans la caractérisation et l'analyse des dimensions tropologiques du discours se trouve dans The Princeton Encyclopedia of Poetry and Poetics, publiée sous la direction d'Alex Preminger et al., Princeton, 1965. En conservant ce système tropologique quaternaire, nous sommes également assurés de résister à toute conception binaire, langage $v s$. style. De riches possibilités combinatoires peuvent ainsi être confectionnées à partir des classifications stylistiques binaires. Nous ne sommes après tout pas obligés de suivre Jakobson quand il réduit l'historiographie du XIX $X^{\mathrm{e}}$ siècle à l'opposition entre une tradition romantico-poético-métaphorique d'une part, et une tradition réalistico-prosaïco-métonymique d'autre part. En fait, ces deux traditions obéissent aux mêmes conventions rhétoriques: tout est affaire de stratégies de discours, dans l'usage plus ou moins extensif que chaque écrivain ou penseur décide de faire des quatre grandes figures de style.
} 
les choses différemment. La métaphore est essentiellement un mode de représentation, la métonymie est réductionniste, la synecdoque synthétise, et l'ironie est de l'ordre de la négation.

Venons-en aux exemples. Soit l'expression métaphorique «Mon amour, ma rose. » Elle pose qu'il est adéquat de parler de la personne que l'on aime comme d'une rose, au sens figuré, bien qu'elle ne soit pas cette fleur au sens littéral. En raison de sa beauté, de sa délicatesse, de son caractère précieux, la personne aimée (car ici « mon amour» est pris dans ce sens particulier) possède les attributs de la rose, symbole de l'amour, et en épouse la figure. « Mon amour » partage certaines qualités avec les roses mais, comme personne elle est d'autant plus unique. On ne peut réduire « mon amour » à n'être qu'une rose, comme ce serait le cas dans la métonymie (ou la synecdoque); la rose n'est pas non plus invoquée de manière ironique, pour signifier que « mon amour » est tout le contraire d'une rose.

Passons à la métonymie, par exemple dans « cinquante voiles » pour dire « cinquante navires ». Comme dans « Mon amour, ma rose », deux objets distincts sont comparés, mais cette fois la substitution consiste bien à réduire le navire à l'une de ses composantes, la voile. Ce n'est pas exactement une relation microcosme/macrocosme, car la voile n'est pas sensée ici symboliser une qualité qu'elle partage avec le navire - sinon nous aurions une synecdoque. Il est seulement possible de reconnaître un navire à sa voile, sans laquelle il ne pourrait naviguer. L'entité « navire » est réduite à l'un de ses aspects, ou à l'une de ses fonctions. La relation n'est donc pas, comme dans la métaphore, entre un objet et un autre objet, mais entre le tout ou une partie d'un objet, et une autre partie représentative du même objet. Examinons maintenant « le grondement du tonnerre $»$ : il y a métonymie, puisque nous trouvons rassemblés dans le même syntagme la cause et l'effet, sans que l'on puisse dire que le tonnerre est seulement la cause, puisqu'il est aussi un son ( « j'entends le tonnerre »), un son qui prend ici la forme particulière d'un grondement: le tonnerre gronde, et devient ce grondement. Comme l'ont compris Vico, Hegel et Nietzsche, de tels concentrés métonymiques permettent de peupler la réalité phénoménale de forces invisibles mais agissantes: les agents et les causes sont distingués des actions et des effets par l'effet d'une opération purement linguistique, qui pourvoit ce faisant aux 


\section{Labyrinthe, $n^{\circ} 33$}

catégories conceptuelles (agents, causes, esprits, essences) nécessaires à la réflexion civilisée de la théologie, de la science et de la philosophie.

La relation qui existe entre le tout et la partie (ou entre la cause et l'effet) n'est pas forcément une question d'opposition entre microscopie et macroscopie, l'opposition étant plutôt entre intrinsèque et extrinsèque. Dans la synecdoque, il est possible de construire deux « parties » sans que l'une soit la réplique miniature de l'autre, et sans que le tout soit équivalent à la somme des parties. Prenons, par exemple, le syntagme suivant: « Il a le cœur sur la main. » Nous avons bien ici une métonymie, au sens ou une partie du corps, le cœur, renvoie au tout de la personne physique. Toutefois, «cœur» doit ici être entendu de manière figurée, non pas comme un organe musculaire, mais comme un trait de personnalité, ou une vertu qui, dans l'imaginaire occidental conventionnel, a son siège dans le cœur. Il entretient donc avec la personne une relation différente de celle que la voile entretient avec le navire. Le cœur est un microcosme, il symbolise un trait de caractère de l'individu, cosmos d'éléments anatomiques et spirituels caractéristiques. Par conséquent, dans « il a le cœur sur la main », la métonymie se double d'une synecdoque. Si nous prenions l'expression au sens littéral, elle deviendrait absurde, laissant entrevoir une personne dotée d'une curieuse anatomie; si nous n'avions qu'une métonymie, l'effet serait incomplet - un peu comme avec l'expression anglaise équivalente he is all heart, qui ne veut pas dire que le corps se réduit à un seul organe ou à une seule fonction corporelle. Pour que l'image soit « parlante », il faut par conséquent qu'elle soit plus qu'une substitution nominale du tout à la partie (comme lorsque « voiles » signifie « navires »), et qu'elle intègre différentes relations logiques dans un même syntagme. En l'occurrence, une totalité (« il ») possède certaines qualités (générosité, compassion...) tellement omniprésentes qu'elles finissent par constituer sa «nature», et non plus seulement une partie de sa personnalité. La construction est bien métonymique, au sens où le cœur relie les différentes autres parties du corps; mais il importe en tant qu'il est l'organe de la générosité. Synecdoque, donc, au sens où « il » est un réseau cohérent d'organes physiques et de qualités spirituelles: tout participe à faire de lui ce qu'il est avant tout, un homme de cœur.

Les trois tropes qui précèdent peuvent ainsi être considérés comme des paradigmes, mis à notre disposition par le langage lui-même, permettant de lever les obstacles cognitifs à la mise en forme pré-conceptuelle de 
l'expérience historique; cette préfiguration précède et permet l'analyse et l'explication. Autrement dit, la pensée peut trouver dans le langage lui-même d'autres paradigmes de captation du réel: la métaphore, qui vise avant tout à représenter le monde, comme dans l'historiographie formiste; la métonymie, qui sert l'approche mécaniciste; et la synecdoque, qui « intègre » chaque élément à la manière organiciste. Ces paradigmes tropologiques privilégient un certain type de relations: de l'objet à un autre objet (métaphore), de la partie à une autre partie d'un même objet (métonymie), ou de la partie au tout de l'objet (synecdoque). Ils induisent des protocoles linguistiques particuliers, selon que l'historien favorise le registre de l'identité (métaphore), de l'extrinsèque (métonymie) ou de l'intrinsèque (synecdoque).

À ces tropes pour «naïfs »-car on ne les utiliserait pas si on ne croyait pas dur comme fer dans la capacité du langage figuré à saisir la vraie nature les choses -, l'ironie offre un contrepoint que je qualifierais de « sentimental», au sens que Schiller donne à ce terme, c'est-à-dire « sans naïveté ». L'ironie est une stratégie discursive essentiellement dialectique, dans la mesure où elle consiste à dire l'inverse de ce que l'on pense afin que l'autre comprenne l'inverse de ce qu'on dit. Elle prend la forme d'un énoncé absurde (ou catachrèse, du grec « faire mauvais usage »), tout en s'efforçant de laisser entendre la distance qui existe entre ce que l'on formule, d'une part, et la réalité et/ou ce que l'on pense réellement, d'autre part. Le tour de passe-passe préféré de l'ironiste consiste à faire précéder cette pseudo-vérité d'une aporie (du grec aporia, doute), par laquelle il injecte par avance du soupçon sur le caractère non crédible de son propre énoncé; ce tour est employé aussi bien dans la fiction que dans les « histoires » rapportées sur le ton du scepticisme et du relativisme.

Parce qu'elle consiste en une formulation inversée, l'ironie contient une intention tacite ou implicite, et suppose donc que le lecteur ou l'auditeur soit prévenu, ou ait l'ouïe assez fine pour reconnaître ce que la caractérisation a d'absurde, et en déduise le contraire du libellé, qu'il s'agisse d'une métaphore, d'une métonymie ou d'une synecdoque. Ainsi, « il a le cœur sur la main », énoncé sur un ton sarcastique à propos d'une personne qui, à l'évidence, ne mérite pas ce compliment, devient une synecdoque ironique. 


\section{Labyrinthe, $n^{\circ} 33$}

Il saute aux yeux que l'ironie est en ce sens un métatrope, qui joue sur la conscience du caractère problématique du langage et met en évidence les possibilités de retourner la rhétorique contre elle-même. De manière parodique, l'ironie révèle l'absurdité de notre croyance dans la capacité du langage à révéler le monde tel qu'il est. Comme l'a noté Kenneth Burke, le procédé est « dialectique », moins du fait d'une certaine appréhension de l'histoire que d'une certaine appréhension du langage figuré qui, manifestement, obscurcit notre perception des choses au lieu de la clarifier. Le discours se replie sur lui-même afin de souligner clairement les limites de notre entendement; c'est pourquoi l'on regarde souvent les propositions ironiques comme les plus sophistiquées et les plus « réalistes ». Elles semblent en effet manifester le libre exercice de notre pensée critique, qui ne s'en laisse pas conter, et n'hésite pas à questionner notre propre compréhension des processus historiques.

Par conséquent, nous disposons avec le trope de l'ironie d'un paradigme intellectuel radicalement différent, parce que porteur d'une dimension autocritique qui s'applique non seulement aux caractérisations de l'expérience historique, mais aux phénomènes linguistiques eux-mêmes. Il est donc l'expression conventionnelle d'un scepticisme de la pensée et d'un relativisme de la morale. Comme possible paradigme de mise en forme du réel, l'ironie est par essence hostile aux protocoles " naïfs " que sont les autres grands modèles explicatifs (formiste, mécaniciste et organiciste). Et sa traduction sur le plan narratif, la satire, ne peut que s'opposer point par point aux modes de représentation correspondants du progrès humain dans l'histoire (romance, comédie, tragédie). Si l'on s'essayait à en déduire une projection existentielle complètement ironique, la vision du monde qui en résulterait serait immanquablement transidéologique, au sens où l'ironie peut être utilisée à l'appui de n'importe quel projet politique. L'ironiste peut s'en prendre au conformisme social aussi bien qu'aux marchands de sable qui proposent de renverser le statu quo. Et l'extrême gauche se sert aussi de l'ironie, pour clouer au pilori ses opposants centristes et conservateurs. Toutefois, lorsqu'elle fonde et oriente toute une vision du monde, l'ironie dissout la possibilité même d'un engagement politique constructif. Devant ce qu'il perçoit comme la bêtise de la civilisation, ou l'absurdité de la condition humaine, l'ironiste arrive à la conclusion simple que « le monde est fou ». Depuis sa hauteur 


\section{Poétiques de l'histoire}

mandarinale, il n'a que dédain amusé pour ceux qui n'ont pas renoncé à percer les mystères de la société.

\section{Les étapes de la conscience historique au XIX ${ }^{\mathrm{e}}$ siècle}

La théorie des tropes, envisagée comme une théorie générale du langage poétique, permet de décrire précisément les écoles qui ont dominé l'historiographie du XIX $x^{\mathrm{e}}$ siècle. En outre, elle m'aide à analyser les structures profondes de l'imaginaire historique de la période, qui constitue un cycle complet. De fait, on peut estimer que chaque mode d'écriture de l'histoire marque une étape dans l'évolution d'une tradition rhétorique : au XIX ${ }^{\mathrm{e}}$ siècle, le modus intellegendi et operandi des historiens fut majoritairement métaphorique, puis métonymique; vint ensuite le tour de la synecdoque, finalement supplantée par l'ironie, c'est-à-dire le relativisme absolu vis-à-vis de toute connaissance.

La première séquence historiographique s'ouvrit avec la crise des Lumières. La conception ironique de l'histoire défendue par des penseurs comme Voltaire, Gibbon, Hume, Kant et Robertson, rencontra une vive résistance: Rousseau (j'entends, le Rousseau préromantique), Justus Möser, Edmund Burke, les poètes suisses de la nature, les auteurs du Sturm und Drang et surtout Herder revendiquaient au contraire un idéalisme « naïf ». Leur critique, parfois floue, de la génération précédente variait d'un auteur à l'autre; mais tous ressentaient la même antipathie vis-à-vis du rationalisme « détaché » et desséchant des Lumières, et prônaient au contraire une approche « empathique » et « humanitaire » des événements historiques - une attitude considérée comme déplacée, et traitée avec condescendance et mépris par leurs aînés. Le schisme historiographique qui s'ensuivit raviva l'intérêt pour les questions théoriques, de sorte que, dans la première décennie du XIX ${ }^{\mathrm{e}}$ siècle, « le problème de la connaissance historique » se trouva au centre des débats philosophiques.

Plus qu'aucun autre, Hegel fut le philosophe qui saisit les racines et les enjeux profonds de ce problème, qui devait l'occuper pendant les deux décennies qui séparent la publication de La Phénoménologie de l'esprit (1806) de celle de la Philosophie de l'histoire (1830-1831). Hegel identifia correctement la raison fondamentale du schisme: la différence irréductible entre une approche ironique et une approche métaphorique 


\section{Labyrinthe, $n^{\circ} 33$}

de l'histoire. En outre, son propre système contenait des arguments rationnels justifiant l'adoption d'une nouvelle approche s'organisant autour de la synecdoque.

Dans le même temps, le rationalisme des Lumières s'offrait une deuxième jeunesse sous la forme de l'organicisme triomphant. Il revenait à Auguste Comte, dont le Cours de philosophie positiviste commença à paraître en 1830, de célébrer les noces des théories mécanicistes des Lumières et de la conception organique du processus historique; Comte consacra aussi le mode narratif de la comédie, au détriment de la satire teintée de pessimisme cultivée par les Lumières finissantes.

Par conséquent, le premier tiers du XIX ${ }^{\mathrm{e}}$ siècle vit coexister trois grandes « écoles » historiographiques: « le Romantisme », " l'Idéalisme », et « le Positivisme». Et, bien que les tenants de chaque théorie soient en désaccord sur la «bonne » façon d'étudier et d'expliquer l'histoire, ils s'accordaient pour répudier l'attitude ironique, typique du rationalisme des Lumières. Cette commune antipathie ne fut pas pour rien dans l'enthousiasme pour les études historiques que nous constatons durant cette période; en outre, elle explique partiellement l'aplomb qui émane des écrits des années 1800-1830, par-delà les divergences «méthodologiques » des historiens.

Elle explique aussi la tonalité des débats pendant la phase suivante, qui va des années 1830 aux années 1870 ; période de maturation classique, marquée par de solides discussions théoriques, mais aussi par une production soutenue de sommes d'érudition. Quatre auteurs sortent du lot, et ce sont les quatre grands « maîtres » de l'historiographie du XIX ${ }^{\mathrm{e}}$ siècle: Michelet, Ranke, Tocqueville et Burckhardt.

Le trait le plus frappant des historiens de cette période est leur degré de conscience théorique. Non contents d'explorer et de représenter le passé, ils sont mus par une haute aspiration: offrir une perspective dynamique de l'histoire qui soit aussi « objective » que l'étude de la nature par les scientifiques, et aussi « réaliste » que le sens pragmatique des hommes d'État ayant en charge la fortune de leur nation. Logiquement, le débat tendit à porter sur les critères permettant de juger du sérieux des différentes conceptions véritablement « réalistes » de l'histoire. En cela, nos historiens ne différaient pas de leurs contemporains romanciers: leurs tableaux historiques se voulaient affranchis à la fois de l'abstraction des Lumières et des illusions des Romantiques. Mais comme leurs épigones 
Scott, Balzac, Stendhal, Flaubert et Goncourt, ils ne réussirent qu'à engendrer autant de variantes du « réalisme » qu'il existait de façons de refaire le monde à l'aide du langage figuré. Partis à l'assaut du réalisme « ironique » des Lumières, ils fourbirent un arsenal de « réalismes » hétéroclite à la mesure... de la diversité des usages possibles de la métaphore, de la métonymie, et de la synecdoque! De fait, je n'aurais pas de mal à démontrer que le "réalisme historique » revendiqué par Michelet, Tocqueville, et Ranke, n'était guère plus que l'invention d'un style personnel, certes à partir d'une perspective critique, mais selon des voies rhétoriques toutes tracées. Quant à Burckhardt, force est de constater qu'il retomba plus d'une fois dans l'ornière de l'ironie, dont le « réalisme » était pourtant censé émanciper la conscience historique de ses contemporains.

Cette exfoliation en modes historiographiques différents fut accompagnée et dans une large mesure causée par un regain de la réflexion sur la philosophie de l'histoire. La seconde période (1830-1870) vit la multiplication des attaques contre le système hégélien, sans qu'aucun philosophe ne réussisse à faire avancer d'un cran sa réflexion sur la conscience historique. L'exception ici est bien sûr Karl Marx, qui tenta de combiner les stratégies synecdochiques de Hegel et les stratégies métonymiques propres à l'économie politique de son temps, afin de produire une vision de l'histoire qui soit à la fois « dialectique » et « matérialiste».

Le concept de matérialisme dialectique, à la fois « historique» et « mécaniciste », représente (au moins pour le XIX ${ }^{\mathrm{e}}$ siècle) la tentative la plus cohérente de transformer l'étude de l'histoire en science. En outre, personne n'a autant que Marx analysé la relation entre la conscience historique et les conditions objectives d'existence historique. Dans l'œuvre de Marx, la théorie et la pratique de l'historien sont intimement liées aux théories et aux pratiques sociales dominantes - elles prennent racine dans une société particulière. Plus qu'aucun autre penseur, Marx était sensible aux implications idéologiques de toutes les conceptions de l'histoire sans exception, y compris celles se réclamant officiellement $\mathrm{du}$ « réalisme »; et, bien que sa propre conception fût tout sauf ironique, il ne se priva pas de découvrir le pot aux roses. Ce faisant, il contribua certainement à pousser l'histoire sur la pente de l'ironie, qui devait caractériser la dernière phase historiographique du XIX ${ }^{\mathrm{e}}$ siècle. 


\section{Labyrinthe, $n^{\circ} 33$}

Au vrai, les années 1870-1900 auraient été celles d'une « crise de l'historicisme » même sans Marx. Les succès de la génération précédente avaient à eux seuls de quoi précipiter la crise de confiance et la plongée dans l'ironie de la conscience historique. Le problème, avec les grands historiens " réalistes » qui n'avaient que « l'objectivité scientifique » à la bouche, c'était qu'ils avaient formulé des hypothèses également approfondies et plausibles, mais mutuellement exclusives, sur les mêmes événements historiques: il y avait de quoi semer le doute. Il est déjà perceptible sous la plume de Burckhardt, dont le scepticisme s'accompagne d'un ton cynique et désabusé quant à la possibilité même de pouvoir vraiment connaître le fond des choses.

L'équivalent philosophique de ce spleen d'historien esthétisant se trouve bien sûr dans l'œuvre de Friedrich Nietzsche. Mais là où, chez Burckhardt, le pessimisme fin-de-siècle donnait tout au plus une coloration personnelle au « réalisme » qu'il continuait de revendiquer, Nietzsche jugea que l'ironie était un problème sérieux. Il voyait en elle le symptôme d'une décadence spirituelle, dont l'humanité ne pouvait guérir que si l'on délivrait la conscience malheureuse de l'historien d'un défi impossible à relever: transcender tous les obstacles et représenter le monde de manière « réaliste ».

Dans ses œuvres philosophiques de jeunesse, Nietzsche fit sien le problème de la conscience ironique de ses contemporains; il ne pouvait dès lors ignorer les formes de conceptualisation historique sur lesquelles cette dernière prospérait. Bien que son état d'esprit et son calcul fussent différents de ceux de Hegel, Nietzsche se donna le même objectif: en finir avec l'ironie, sans retomber dans les illusions du Romantisme « naïf ». En fait, Nietzsche opéra un retour à la conception romantique de l'histoire, dans la mesure où il tenta d'assimiler la réflexion historique à une démarche artistique. La métaphore jouait un rôle éminent dans cette stratégie théorique. À le lire, l'historiographie devait se doter d'une théorie délibérément métahistorique et d'un horizon « supra-historique ». Aussi, Nietzsche incita l'historien à considérer le champ historique à la lumière d'une réflexivité métaphorique. C'est bien là, de son fait, l'ironie de l'histoire: son propos n'était que métaphoriquement ironique. Dans la pensée nietzschéenne, qui relevait d'une approche poétique de la réalité, la conscience psychologique confrontée à l'histoire restait une énigme à analyser. En attendant, Nietzsche, comme Marx, contribuait à aggraver 
un peu plus la crise de l'historicisme, à laquelle la pensée historique de l'époque finit par succomber tout entière.

C'est pour répondre à cette crise que Benedetto Croce entreprit sa monumentale étude sur les structures profondes de la conscience historique. Comme Nietzsche, Croce voyait bien que la crise de l'historicisme reflétait avant tout le triomphe du scepticisme ironique. Et, comme Nietzsche, il avait l'espoir de purger la pensée historique de toute ironie en la mettant sur le même plan que l'art. Le paradoxe est que, ce faisant, il fut conduit à élaborer une conception particulièrement ironique de l'art, de sorte que, par la logique même de l'assimilation, il ne réussit finalement qu'à mettre un peu plus devant l'évidence de leur condition les historiens réfléchissant à leur pratique: l'exigence accrue de réflexivité les condamnait une fois encore au scepticisme ironique. Par la suite, Croce essaya de le faire sortir de cette mauvaise passe, en assimilant cette fois l'histoire à la philosophie. Mais il ne parvint qu'à historiciser cette dernière, et donc à rendre les philosophes aussi ironiquement conscients de leurs limites que l'étaient les historiens.

De ce point de vue, l'évolution de la philosophie de l'histoire depuis Hegel jusqu'à Croce, en passant par Marx et Nietzsche, a suivi une trajectoire semblable à celle de l'historiographie, de Michelet à Burckhardt en passant par Ranke et Tocqueville: rien d'étonnant, puisqu'on avait les mêmes dynamiques conceptuelles. Seul l'ordre des séquences paradigmatiques différait. L'important est que la philosophie de l'histoire était arrivée au même point que l'historiographie dans le dernier tiers $\mathrm{du} \mathrm{XIX}^{\mathrm{e}}$ siècle. Cette condition ironique ressemblait à s'y méprendre à celle de la fin du XVIII ${ }^{\mathrm{e}}$ siècle, bien que la philosophie de l'histoire lui ait insufflé un haut degré de sophistication, et que l'historiographie de l'époque ait porté l'histoire à des sommets d'érudition. 